ESAIM: M2AN

Vol. 40, No 4, 2006, pp. 653-687

DOI: $10.1051 / \mathrm{m} 2 \mathrm{an}: 2006028$
ESAIM: Mathematical Modelling and Numerical Analysis

www.edpsciences.org/m2an

\title{
STUDY OF A THREE COMPONENT CAHN-HILLIARD FLOW MODEL
}

\author{
FRANCK BOYER $^{1}$ AND CÉline LAPUERTA ${ }^{2}$
}

\begin{abstract}
In this paper, we propose a new diffuse interface model for the study of three immiscible component incompressible viscous flows. The model is based on the Cahn-Hilliard free energy approach. The originality of our study lies in particular in the choice of the bulk free energy. We show that one must take care of this choice in order for the model to give physically relevant results. More precisely, we give conditions for the model to be well-posed and to satisfy algebraically and dynamically consistency properties with the two-component models. Notice that our model is also able to cope with some total spreading situations. We propose to take into account the hydrodynamics of the mixture by coupling our ternary Cahn-Hilliard system and the Navier-Stokes equation supplemented by capillary force terms accounting for surface tension effects between the components. Finally, we present some numerical results which illustrate our analysis and which confirm that our model has a better behavior than other possible similar models.
\end{abstract}

Mathematics Subject Classification. 35B35, 35K55, 76T30.

Received: October 18, 2005. Revised: March 31, 2006.

\section{INTRODUCTION}

Multi-component flows are frequently encountered in engineering applications, like for instance nuclear safety studies which are the industrial context of this work performed for the French "Institut de Radioprotection et de Sûreté Nucléaire". Diffuse interface models (see for instance the review in [2]) are now widely used to describe diphasic systems (see $[6,15,28]$ and Sect. 1). In these models, the thickness of the interface between the two phases is supposed to be very small but positive. Each phase is then represented by a smooth function called the order parameter (which is often taken to be the volumic fraction of the component in the mixture). The evolution of the system is then driven by the gradient of a total free energy which is the sum of two terms: a bulk free energy term with a "double-well" shape and a capillary term depending on the gradient of the order parameter and which accounts for the energy of the interface, that is the surface tension.

It was shown by some authors $[5,6,15,16,23]$ that one can couple diffuse interface models with the NavierStokes equation in order to cope with the hydrodynamics of such miscible or immiscible mixtures. The final model takes into account the main phenomenon of such flows and is well-suited to numerical simulations since there is no need to follow the interface or to solve free boundary problems.

\footnotetext{
Keywords and phrases. Multicomponent flows, Cahn-Hilliard equations, stability.

${ }^{1}$ Laboratoire d'Analyse, Topologie et Probabilités, 39 rue F. Joliot Curie, 13453 Marseille Cedex 13, France.

fboyer@cmi.univ-mrs.fr

2 Institut de Radioprotection et de Sûreté Nucléaire, DPAM/SEMIC/LMPC, BP 3, 13115 Saint-Paul-lez-Durance, France.
}

(C) EDP Sciences, SMAI 2006 
Generalizations of diffuse interface models to any number of components have been recently introduced and studied for instance in $[9,10,12,13,21]$. Numerical methods were also proposed and used to simulate multi-phase transitions for instance in $[3,4,7]$. Kim and coworkers have also studied how to generalize the coupling between such multi-component Cahn-Hilliard models and the Navier-Stokes equations and how to implement efficient numerical solvers (see $[18,20,21])$.

In this paper, we propose to study with more details the diffuse interface models which are well-suited (when coupled with a momentum balance equation) for the simulation of incompressible flows made of three immiscible phases, with no phase change. As in the references cited above, our study is based on the CahnHilliard approach. One of the main feature of the present work is that, thanks to a relevant choice of the free energy, our model coincides exactly with the diphasic Cahn-Hilliard model (described in Sect. 1, see also [8,22]) when only two phases are present in the mixture. In this case, we say that the model is algebraically consistent with binary systems. Furthermore, we are able to prove that our model is well-posed and that it is dynamically consistent with binary systems. This last property is an asymptotic stability property of binary solutions inside the ternary ones. These consistency properties ensure that non-physical apparition of one phase inside the interface between the other two will not occur as it is confirmed in numerical simulations given at the end of the paper. We want to lay stress on the fact that our study is only devoted to the modelling of immiscible phases without phase change and is not necessarily relevant to more general situations like ternary spinodal decomposition.

In order to describe the hydrodynamics of the mixture, we follow previous studies in the field in order to perform the coupling between our particular ternary Cahn-Hilliard model and the Navier-Stokes equation for an incompressible flow (in the so-called quasi-incompressible framework see [23]). These equations are defined on the whole domain (there is no jump conditions) and the interfacial surface tension forces are naturally taken into account through volumic capillary forces (see [19]). The final fully coupled model is very well adapted to numerical simulations since interface tracking and surface forces discretization are not needed, just like in the diphasic case.

The outline of the paper is the following. In Section 1, a brief introduction to the Cahn-Hilliard diphasic model is given. In the second one, we present the general construction of ternary models based on a given but still undetermined free energy for the system. Then we state Theorem 2.4 which says that, under suitable assumptions on this energy, there exists a unique global weak solution to the problem. We want to point out here that the assumptions needed to prove this last result are more general than the usual ones in the literature and allow us to apply this well-posedness result to all the particular models we study in the remaining of the paper. Notice, in particular, that we are able to cope with some total spreading situations (see for instance Rem. 3.3 and Sect. 5.3.4). To our knowledge, this is a new feature compared to other studies.

In the third section we prove that the bulk free energy in the model has to fit a particular form (Thm. 3.2) in order for the system to be physically relevant. We study particular examples of such suitable bulk free energies in Sections 3.2 and 3.3, and we provide a complete analysis for each of them. We also prove that, under suitable conditions, these particular models are dynamically consistent with diphasic systems (Thms. 3.8 and 3.9). This ensures the stability of binary interfaces inside a ternary system.

Fourth section is devoted to the proof of our well-posedness result stated at the end of Section 2. Finally, Section 5 is dedicated to numerical simulations. Some results are presented in the classical case of a lens located at the interface between the other two components (see $[25,26]$ ). The contact angles between the phases and the pressure jumps are correctly computed. Moreover, our theoretical results are illustrated by some numerical evidences that non consistent models as well as some other models in the literature (see [13,21]) may lead to unsatisfactory results compared to our models. We conclude this section with some results obtained by solving the whole Navier-Stokes/Cahn-Hilliard system in order to simulate the rising of a bubble across a liquid/liquid interface. Our results are in agreement with a theoretical predictive criterion on the volume of the bubble given in [14] to determine whether or not the bubble will cross the liquid/liquid interface. This proves that capillarity effects are correctly captured by our model. 


\section{The two-Component CAhn-Hilliard Model}

We give here a brief overview of the Cahn-Hilliard model for two non-miscible constituents. The domain $\Omega$ is an open bounded, connected, subset of $\mathbb{R}^{d}$ with $d=2$ or $d=3$, with a sufficiently smooth boundary.

The Cahn-Hilliard approach consists in assuming that the interface between the two phases in the system has small but finite width $\varepsilon$. Then, the composition of the mixture is described at each point by the value of an order parameter $c$ which is typically the concentration of one of the constituent in the mixture. The value of the order parameter is 0 or 1 where only one of the phases is present and varies rapidly but smoothly across the interface.

Moreover, the evolution of the system is driven by the minimization of a free energy under the constraint of mass conservation of each phase that is $\int_{\Omega} c \mathrm{~d} x=$ constant. The expression of the free energy of the mixture depending on two parameters $\varepsilon$ (the interface width) and $\sigma$ (the surface tension) can be written as follows

$$
\mathcal{F}_{\sigma, \varepsilon}^{\operatorname{diph}}(c)=\int_{\Omega} 12 \frac{\sigma}{\varepsilon} c^{2}(1-c)^{2}+\frac{3}{4} \sigma \varepsilon|\nabla c|^{2} \mathrm{~d} x
$$

The first term (the bulk free energy) is non-negative and has a classical double-well structure with two minima for $c=0$ and $c=1$ corresponding to the two pure phases, whereas the second term (the capillary term) tends to penalize the interfacial zone. The equilibrium shape of infinite plane interfaces, in one dimension, can be explicitly computed by solving the $1 \mathrm{D}$ problem

$$
\left\{\begin{array}{l}
-\frac{3}{2} \sigma \varepsilon c_{0}^{\prime \prime}+24 \frac{\sigma}{\varepsilon} c_{0}\left(1-c_{0}\right)\left(1-2 c_{0}\right)=0 \\
\lim _{+\infty} c_{0}=1 \\
\lim _{-\infty} c_{0}=0
\end{array}\right.
$$

whose solution is, up to a translation,

$$
c_{0}(x)=0.5\left(1+\tanh \left(\frac{2 x}{\varepsilon}\right)\right) .
$$

Hence, we can see that $\varepsilon$ can be considered as the characteristic size of the transition layer between the two stable states $c=0$ and $c=1$ which is called the interface. We can now compute the total energy contained in such an interfacial shape and we find that

$$
\int_{\mathbb{R}} \mathcal{F}_{\sigma, \varepsilon}^{\operatorname{diph}}\left(c_{0}\right) \mathrm{d} x=\sigma,
$$

so that $\sigma$ is exactly the surface tension contained in the interface per surface unit. A very important feature of the Cahn-Hilliard approach is that the two relevant physical parameters which are $\sigma$ and $\varepsilon$ can be imposed independently one from each other. In particular, it is possible for numerical purposes to choose $\varepsilon$ depending on the mesh size we use without modifying the surface tension $\sigma$ contained in the model.

Once we have defined this energy $\mathcal{F}_{\sigma, \varepsilon}^{\text {diph }}$, we can describe the time evolution of the mixture, thanks to the Cahn-Hilliard equation

$$
\left\{\begin{array}{l}
\frac{\partial c}{\partial t}-\nabla \cdot(M \nabla \mu)=0 \\
\mu=\frac{\delta \mathcal{F}_{\sigma, \varepsilon}^{\operatorname{diph}}}{\delta c}=-\frac{3}{2} \sigma \varepsilon \Delta c+24 \frac{\sigma}{\varepsilon} c(1-c)(1-2 c)
\end{array}\right.
$$

which is nothing but the flow along the gradient of the functional $\mathcal{F}_{\sigma, \varepsilon}^{\text {diph }}$ with respect to the $\mathrm{H}^{-1}(\Omega)$ inner product ensuring the mass conservation property. The functional derivative of the energy with respect to the 
order parameter, called $\mu$, is the chemical potential of the mixture and the coefficient $M$ (which may depend on $c$ ) is a diffusion coefficient called mobility. The first natural boundary condition for this equation is the homogeneous Neumann boundary condition for $\mu$ which ensures that there is no diffusion through the boundary of the domain. Another boundary condition is necessary to complete the system. It is often taken to be the homogeneous Neumann condition for the order parameter $c$ which models the fact that the interface is supposed to be orthogonal to the boundary of the domain. If one wants to model more general contact angles on the boundary of the domain, it is possible to apply non-homogeneous and non-linear Neumann conditions to this problem (see [16]).

Remark 1.1. Note that, $c$ being the concentration of one of the two phases in the mixture, it must satisfy $0 \leq c \leq 1$. Unfortunately, it is well-known that this property does not hold in general for the solutions of (2) (we recall that there is no maximum principle for such fourth-order parabolic equations). This is a drawback of this kind of models that we will also encounter for ternary systems in the sequel of the paper. One way to ensure that the values of the concentration $c$ stay between 0 in 1 , is to allow degenerate mobility $\left(M(c)=c^{2}(1-c)^{2}\right.$ for instance) or non-smooth bulk free energies (e.g. with logarithmic singularities in 0 and 1 ). In that case, the analysis is more intricate (see e.g. $[3,5]$ ) and in particular uniqueness of solutions is not known. We concentrate in this paper on non-degenerate mobilities and smooth bulk free energies.

\section{Three-COmponent Cahn-Hilliard models}

In this section, we propose a general description of ternary Cahn-Hilliard models.

\subsection{General framework}

The aim of this section is to propose a well-suited generalization of the diffuse interface model presented above in the case of ternary systems made of three immiscible components. Like for the binary model, the given physical parameters are the three constant surface tensions $\sigma_{12}, \sigma_{13}$ and $\sigma_{23}$ between two of the three components and the interfacial width $\varepsilon>0$, which is supposed to be the same for the three kinds of interfaces. This assumption is not restrictive since, in practical situations, the parameter $\varepsilon$ is chosen so that the interfacial zone contains at least 2 or 3 cells of the mesh. The key-point here is that, this artificial modification of the interface width can be performed without changing the surface tensions contained in the model.

As a starting point, we describe a ternary system by using three order parameters, $c_{1}, c_{2}$ and $c_{3}$, each of them being the concentration of one the components in the mixture. Since we only consider perfect mixtures, these three unknowns are linked through the relationship

$$
c_{1}+c_{2}+c_{3}=1
$$

so that, admissible states will belong to the hyperplane

$$
\mathcal{S}=\left\{\left(c_{1}, c_{2}, c_{3}\right) \in \mathbb{R}^{3}, \quad c_{1}+c_{2}+c_{3}=1\right\} .
$$

We recall that the physical condition $c_{i} \geq 0$ can not be guaranteed in our framework (see Rem. 1.1).

We will now describe the Cahn-Hilliard energy for such a system. In view of the diphasic case, it is natural to look for such energy as the sum of a bulk free energy $F\left(c_{1}, c_{2}, c_{3}\right)$, that is a kind of "triple-well" structured non-convex function to be determined, and capillary terms $\left|\nabla c_{i}\right|^{2}$. We postulate that the free energy can be written as follows

$$
\mathcal{F}_{\Sigma, \varepsilon}^{\text {triph }}\left(c_{1}, c_{2}, c_{3}\right)=\int_{\Omega} \frac{12}{\varepsilon} F\left(c_{1}, c_{2}, c_{3}\right)+\frac{3}{8} \varepsilon \Sigma_{1}\left|\nabla c_{1}\right|^{2}+\frac{3}{8} \varepsilon \Sigma_{2}\left|\nabla c_{2}\right|^{2}+\frac{3}{8} \varepsilon \Sigma_{3}\left|\nabla c_{3}\right|^{2} \mathrm{~d} x,
$$

where $\boldsymbol{\Sigma}=\left(\Sigma_{1}, \Sigma_{2}, \Sigma_{3}\right)$ is a triple of constant parameters to be determined. Notice that the coefficients 12 and $\frac{3}{8}$ are natural in view of the diphasic case (1). Note that, in full generality, we may also have considered 
capillary cross terms of the form $\nabla c_{i} \cdot \nabla c_{j}$. Actually, it can be easily shown that the consistency properties we will look for in the remaining of this paper can only hold if the capillary terms are chosen as in (5) with possibly a supplementary term $\left|\nabla\left(c_{1}+c_{2}+c_{3}\right)\right|^{2}$ (containing $\nabla c_{i} \cdot \nabla c_{j}$ terms). Nevertheless, this latter term does not enter the final model since $\mathbf{C}$ is constrained to lie in $\mathcal{S}$.

Most of the work in Section 3 will be to determine how to choose the bulk free energy $F$ and the parameters $\Sigma_{i}$ in order for the model to fit with the prescribed surface tensions $\sigma_{12}, \sigma_{13}$ and $\sigma_{23}$ and to be consistent with the two-component situation. First, let us derive from (5) the general evolution equation satisfied by the order parameters.

Notation. From now on, we will note $\mathbf{C}=\left(c_{1}, c_{2}, c_{3}\right)$ and $\boldsymbol{\mu}=\left(\mu_{1}, \mu_{2}, \mu_{3}\right)$.

The time evolution of $\mathbf{C}$ is governed by the gradient of the energy $\mathcal{F}_{\boldsymbol{\Sigma}, \varepsilon}^{\text {triph }}$ with respect to the $\mathrm{H}^{-1}(\Omega)$ inner product under the additional constraint (3), which has to hold everywhere at any time. In order to ensure this last constraint, we use a Lagrangian multiplier $\beta$ (see [12]). The system of equations is written for $i=1,2,3$

$$
\left\{\begin{aligned}
\frac{\partial c_{i}}{\partial t} & =\nabla \cdot\left(M_{i} \nabla \mu_{i}\right) \\
\mu_{i} & =\frac{\delta \mathcal{F}_{\boldsymbol{\Sigma}, \varepsilon}^{\text {triph }}}{\delta c_{i}}+\beta=-\frac{3}{4} \varepsilon \Sigma_{i} \Delta c_{i}+\frac{12}{\varepsilon} \partial_{i} F(\mathbf{C})+\beta
\end{aligned}\right.
$$

To eliminate $\beta$, we write the equation satisfied by $S=c_{1}+c_{2}+c_{3}$, and we want $S \equiv 1$ to be a solution to this equation. We easily see that this can only happen if we postulate that

$$
M_{0}=M_{1} \Sigma_{1}=M_{2} \Sigma_{2}=M_{3} \Sigma_{3} .
$$

Under this assumption, the sum $S$ satisfies

$$
\frac{\partial S}{\partial t}=\nabla \cdot\left(M_{0} \nabla\left(-\frac{3}{4} \varepsilon \Delta S+\beta\left(\frac{1}{\Sigma_{1}}+\frac{1}{\Sigma_{2}}+\frac{1}{\Sigma_{3}}\right)+\frac{12}{\varepsilon \Sigma_{1}} \partial_{1} F(\mathbf{C})+\frac{12}{\varepsilon \Sigma_{2}} \partial_{2} F(\mathbf{C})+\frac{12}{\varepsilon \Sigma_{3}} \partial_{3} F(\mathbf{C})\right)\right) .
$$

Now we see that $S \equiv 1$ is solution of (7) if and only if the Lagrange multiplier is defined by

$$
\beta=-\sum_{i=1}^{3} \frac{4 \Sigma_{T}}{\varepsilon \Sigma_{i}} \partial_{i} F(\mathbf{C})
$$

where $\Sigma_{T}$ is defined by

$$
\frac{3}{\Sigma_{T}}=\frac{1}{\Sigma_{1}}+\frac{1}{\Sigma_{2}}+\frac{1}{\Sigma_{3}}
$$

Using this expression of $\beta$, the ternary system (6) now reads, for $i=1,2,3$

$$
\left\{\begin{aligned}
\frac{\partial c_{i}}{\partial t} & =\nabla \cdot\left(\frac{M_{0}}{\Sigma_{i}} \nabla \mu_{i}\right), \\
\mu_{i} & =\frac{4 \Sigma_{T}}{\varepsilon} \sum_{j \neq i}\left(\frac{1}{\Sigma_{j}}\left(\partial_{i} F(\mathbf{C})-\partial_{j} F(\mathbf{C})\right)\right)-\frac{3}{4} \varepsilon \Sigma_{i} \Delta c_{i} .
\end{aligned}\right.
$$

Finally, the system (8) is built so that if the initial data is admissible, that is $\mathbf{C}(0) \in \mathcal{S}$, then $\mathbf{C}(t) \in \mathcal{S}$ for any time $t$. Hence, we can eliminate a posteriori one of the three unknowns $c_{1}, c_{2}$ or $c_{3}$, which can be recovered using the constraint (3). In the same way, it is easily seen that the three chemical potentials $\left(\mu_{1}, \mu_{2}, \mu_{3}\right)$ are linked through the relation

$$
\sum_{i=1}^{3} \frac{\mu_{i}}{\Sigma_{i}}=0
$$


Hence, the system (8) reduces to a set of two coupled Cahn-Hilliard equations. This is of course these two equations that are used for numerical computations. Nevertheless, for the theoretical study of the model it is worth working with the full system (8). A very important point here is that, since we only eliminate one of the unknowns a posteriori, the solution we obtain does not depend on the choice of the unknown that we eliminate. This is not true, for instance, for the models developed in $[18,20]$ where the elimination of one of the order parameters is performed in the very beginning of the modeling.

Just like in the diphasic case, we supplement the previous system with Neumann boundary conditions for each of the unknowns $\left(c_{i}, \mu_{i}\right)$ which ensures, in particular, that the volume of each phase is conserved along the evolution.

\subsection{Coercivity of the capillary terms}

We will see later that it may happen (in the so-called total spreading situation, see Sect. 5.3.4) that one of the coefficients $\Sigma_{i}$ appearing in (5) is negative. That is the reason why we want to pay a particular attention to be able to perform the analysis of the system (8) without assuming the positivity of the $\Sigma_{i}$. Nevertheless, for the system to be well-posed it is clear that the total free energy (5) has to be, at least, bounded from below.

This remark leads us to necessary and sufficient conditions on the $\left(\Sigma_{i}\right)_{i}$ that we give below for the capillary part of the energy to be non-negative. This condition (11) (notice that we do not know if it has any physical meaning) lets us cope with some but not all total spreading situations.

Proposition 2.1. Let $\left(\Sigma_{1}, \Sigma_{2}, \Sigma_{3}\right) \in \mathbb{R}^{3}$. There exists $\underline{\Sigma}>0$ such that for any $n \geq 1$,

$$
\Sigma_{1}\left|\xi_{1}\right|^{2}+\Sigma_{2}\left|\xi_{2}\right|^{2}+\Sigma_{3}\left|\xi_{3}\right|^{2} \geq \underline{\Sigma}\left(\left|\xi_{1}\right|^{2}+\left|\xi_{2}\right|^{2}+\left|\xi_{3}\right|^{2}\right), \forall\left(\xi_{1}, \xi_{2}, \xi_{3}\right) \in\left(\mathbb{R}^{n}\right)^{3}, \text { s.t. } \xi_{1}+\xi_{2}+\xi_{3}=0,
$$

if and only if the following two conditions hold

$$
\begin{gathered}
\Sigma_{1} \Sigma_{2}+\Sigma_{1} \Sigma_{3}+\Sigma_{2} \Sigma_{3}>0, \\
\Sigma_{i}+\Sigma_{j}>0, \quad \forall i \neq j .
\end{gathered}
$$

Proof. First of all, since we are only concerned with triples $\left(\xi_{1}, \xi_{2}, \xi_{3}\right)$ satisfying $\sum_{i=1}^{3} \xi_{i}=0$, we see that we only need to study the case $n=2$. Without loss of generality we can also assume that $\xi_{1}=\left(\begin{array}{l}x \\ 0\end{array}\right)$ and $\xi_{2}=\left(\begin{array}{l}y \\ z\end{array}\right)$. Hence, property (10) holds if and only if the quadratic form

$$
Q(x, y, z)=\Sigma_{1} x^{2}+\Sigma_{2}\left(y^{2}+z^{2}\right)+\Sigma_{3}\left((x+y)^{2}+z^{2}\right),
$$

is definite positive. The matrix associated to this quadratic form is given by

$$
\mathbf{Q}=\left(\begin{array}{ccc}
\Sigma_{1}+\Sigma_{3} & \Sigma_{3} & 0 \\
\Sigma_{3} & \Sigma_{2}+\Sigma_{3} & 0 \\
0 & 0 & \Sigma_{2}+\Sigma_{3}
\end{array}\right)
$$

and it is easily seen, by computing the main minor determinants, that this matrix is definite positive if and only if

$$
\begin{aligned}
\Sigma_{1}+\Sigma_{3} & >0, \\
\left(\Sigma_{1}+\Sigma_{3}\right)\left(\Sigma_{2}+\Sigma_{3}\right)-\Sigma_{3}^{2} & =\Sigma_{1} \Sigma_{2}+\Sigma_{1} \Sigma_{3}+\Sigma_{2} \Sigma_{3}>0, \\
\Sigma_{2}+\Sigma_{3} & >0 .
\end{aligned}
$$

Remarking that

the claim is proved.

$$
\Sigma_{1}+\Sigma_{2}=\frac{\Sigma_{1}^{2}+\left(\Sigma_{1} \Sigma_{2}+\Sigma_{1} \Sigma_{3}+\Sigma_{2} \Sigma_{3}\right)}{\Sigma_{1}+\Sigma_{3}},
$$


We also give, for further use, the following straightforward consequence of the previous result.

Corollary 2.2. Let $\left(\Sigma_{1}, \Sigma_{2}, \Sigma_{3}\right) \in \mathbb{R}^{3}$ such that (11) and (12) hold. There exists $\bar{\Sigma} \geq 0$ such that for any $n \geq 1$ and any $\left(\xi_{1}, \xi_{2}, \xi_{3}\right) \in\left(\mathbb{R}^{n}\right)^{3}$, we have

$$
\Sigma_{1}\left|\xi_{1}\right|^{2}+\Sigma_{2}\left|\xi_{2}\right|^{2}+\Sigma_{3}\left|\xi_{3}\right|^{2} \geq-\bar{\Sigma}\left|\xi_{1}+\xi_{2}+\xi_{3}\right|^{2} .
$$

\subsection{Existence and uniqueness of weak solutions}

We state now the general assumptions needed on the bulk free energy $F$ in order to be able to prove the well-posedness of the system (8):

- We assume that $F$ is of $\mathcal{C}^{2}$ class and satisfies

$$
F(\mathbf{C}) \geq 0, \quad \forall \mathbf{C} \in \mathcal{S},
$$

- There exist $B_{1}, B_{2}>0$ such that

$$
\begin{aligned}
|F(\mathbf{C})| & \leq B_{1}|\mathbf{C}|^{p}+B_{2}, \quad \forall \mathbf{C} \in \mathcal{S} \\
|D F(\mathbf{C})| & \leq B_{1}|\mathbf{C}|^{p-1}+B_{2}, \quad \forall \mathbf{C} \in \mathcal{S}, \\
\left|D^{2} F(\mathbf{C})\right| & \leq B_{1}|\mathbf{C}|^{p-2}+B_{2}, \quad \forall \mathbf{C} \in \mathcal{S},
\end{aligned}
$$

where $p=6$ if $d=3$, and $2 \leq p<+\infty$ if $d=2$.

- There exists $D_{1} \geq 0$ such that

$$
\left(D^{2} F(\mathbf{C}) \xi, \xi\right) \geq-D_{1}\left(1+|\mathbf{C}|^{q}\right)|\xi|^{2}, \quad \forall \mathbf{C} \in \mathcal{S}, \quad \forall \xi \in \mathbb{R}^{3},
$$

where $0 \leq q<4$ if $d=3$ and $0 \leq q<+\infty$ if $d=2$.

Remark 2.3. One can replace (13) by the assumption that $F$ is bounded from below on $\mathcal{S}$ since adding a constant to $F$ does not change the equations (8).

Let us notice that assumptions (13)-(16) are classical in the study of Cahn-Hilliard equations and systems even though some authors study the important case of logarithmic bulk free energies that we do not treat here (see for instance [3]). On the contrary, assumption (17) is more original since in many cases the bulk free energy is supposed to the sum of a convex part and a quadratic non-convex part (see [5,27]) so that assumption (17) is satisfied with $q=0$. We will show in Section 3.3 that it is crucial to be able to cope with more general bulk free energies for which it will be necessary to allow large enough values of $q$ in (17).

More precisely, this assumption (17) is not necessary to prove the existence of solutions to (8) but is needed to establish regularity properties that imply uniqueness of such solutions.

Theorem 2.4. Let $\Omega$ be a bounded smooth domain in $\mathbb{R}^{d}, d=2$ or 3 . Assume that conditions (11), (12) and assumptions (13)-(17) hold and that the mobility $M_{0}$ is a positive constant. For any $\mathbf{C}^{0} \in\left(\mathrm{H}^{1}(\Omega)\right)^{3}$ such that $\mathbf{C}^{0}(x) \in \mathcal{S}$, for almost every $x \in \Omega$, there exists a unique weak solution $(\mathbf{C}, \boldsymbol{\mu})$ of $(8)$ on $[0,+\infty[$ with initial data $\mathbf{C}^{0}$ such that,

$$
\begin{gathered}
\mathbf{C}(t, x) \in \mathcal{S}, \text { for a.e. }(t, x) \in[0,+\infty[\times \Omega, \\
\mathbf{C} \in \mathrm{L}^{\infty}\left(0,+\infty ;\left(\mathrm{H}^{1}(\Omega)\right)^{3}\right) \cap \mathrm{L}_{\text {loc }}^{2}\left(0,+\infty ;\left(\mathrm{H}^{3}(\Omega)\right)^{3}\right) \cap \mathcal{C}^{0}\left(\left[0,+\infty\left[;\left(\mathrm{H}^{1}(\Omega)\right)^{3}\right),\right.\right. \\
\boldsymbol{\mu} \in \mathrm{L}^{2}\left(0,+\infty ;\left(\mathrm{H}^{1}(\Omega)\right)^{3}\right) .
\end{gathered}
$$

In the bidimensional case, this result still holds if we allow the mobility $M_{0}$ in (8) to be a smooth, bounded and non vanishing function of the order parameters $\mathbf{C}$. In the 3D case, existence holds but uniqueness is not known in that case.

We postpone the proof of this result to Section 4 since we want now to discuss how to choose the parameters $\left(\Sigma_{i}\right)_{i}$ and the bulk free energy $F$. 


\section{Algebraichlly and dynamically COnsistent ternary models}

One of the main goals of this work is to give a three immiscible component model which exactly coincides with the two component model described in Section 1 in the case where only two phases are present. We will say that the model is algebraically consistent (see Def. 3.1). Note that this very natural property does not hold for various models studied in the literature (see [13,21] for instance). Actually, it will be shown in Section 5.3 that this consistency property is crucial in view of the numerical simulation of three-component systems.

\subsection{Algebraically consistent models}

Let us first give a list of natural properties that the free energy $\mathcal{F}_{\boldsymbol{\Sigma}, \varepsilon}^{\text {triph }}$ and the Cahn-Hilliard system (8) have to fulfill in order to be physically coherent.

Definition 3.1. We say that the model defined by (5) and (8) is algebraically consistent with the diphasic systems corresponding to the given surface tensions $\sigma_{12}, \sigma_{13}, \sigma_{23}$ respectively if the following properties hold:

(P1) When the component $i$ is not present, that is $c_{i}=0$, the total free energy $\mathcal{F}_{\boldsymbol{\Sigma}, \varepsilon}^{\text {triph }}\left(c_{1}, c_{2}, c_{3}\right)$ of the system has to be exactly equal to the total free energy of the diphasic system containing the two other phases

$$
\begin{aligned}
& \mathcal{F}_{\boldsymbol{\Sigma}, \varepsilon}^{\mathrm{triph}}(c, 1-c, 0)=\mathcal{F}_{\sigma_{12}, \varepsilon}^{\text {diph }}(c), \quad \forall c \in \mathrm{H}^{1}(\Omega), \\
& \mathcal{F}_{\boldsymbol{\Sigma}, \varepsilon}^{\text {triph }}(c, 0,1-c)=\mathcal{F}_{\sigma_{13}, \varepsilon}^{\text {diph }}(c), \quad \forall c \in \mathrm{H}^{1}(\Omega), \\
& \mathcal{F}_{\boldsymbol{\Sigma}, \varepsilon}^{\text {triph }}(0, c, 1-c)=\mathcal{F}_{\sigma_{23}, \varepsilon}^{\operatorname{diph}}(c), \quad \forall c \in \mathrm{H}^{1}(\Omega) .
\end{aligned}
$$

(P2) When the component $i$ is not present in the mixture at the initial time, the component $i$ must not appear during the time evolution of the system. In other words, any admissible solution to the system (8) must satisfy, for any $i \in\{1,2,3\}$,

$$
c_{i}(0)=0 \Longrightarrow c_{i}(t)=0, \forall t \geq 0
$$

All the physical parameters $\varepsilon, M_{0}, \sigma_{12}, \sigma_{13}$ and $\sigma_{23}$ being fixed, our model is completely determined by the expression of the free bulk energy $F$ and of the coefficients $\boldsymbol{\Sigma}=\left(\Sigma_{i}\right)_{i}$ in $(5)$. Assuming that the bulk free energy is smooth we are now able to give a complete characterization of algebraically consistent models according to the above definition.

Theorem 3.2. Let $\sigma_{12}, \sigma_{13}, \sigma_{23}$ be three positive real numbers. The model defined by (5) and (8) is algebraically consistent with the diphasic systems of surface tensions $\sigma_{12}, \sigma_{13}, \sigma_{23}$ respectively if and only if we have

$$
\Sigma_{i}=\sigma_{i j}+\sigma_{i k}-\sigma_{j k}, \forall i \in\{1,2,3\}
$$

and there exist smooth functions $G$ and $H$ such that

$$
\begin{aligned}
F(\mathbf{C})=\sigma_{12} c_{1}^{2} c_{2}^{2}+\sigma_{13} c_{1}^{2} c_{3}^{2}+\sigma_{23} c_{2}^{2} c_{3}^{2}+c_{1} c_{2} c_{3}\left(\Sigma_{1} c_{1}+\right. & \left.\Sigma_{2} c_{2}+\Sigma_{3} c_{3}\right) \\
& +c_{1}^{2} c_{2}^{2} c_{3}^{2} G(\mathbf{C})+\left(c_{1}+c_{2}+c_{3}-1\right) H(\mathbf{C}), \forall \mathbf{C} \in \mathbb{R}^{3}
\end{aligned}
$$

Remark 3.3. In the physical literature (see [25]), the coefficient $S_{i}=-\Sigma_{i}$ defined by (20) is well known and called the spreading coefficient of the phase $i$ at the interface between phases $j$ and $k$. If $S_{i}$ is positive (that is $\Sigma_{i}<0$ ), the spreading is said to be total and if $S_{i}$ is negative, it is said to be partial (see the reference above).

Proof. 1/ Property (P1) applied, for instance, when $c_{1}=0$ implies that

$$
\mathcal{F}_{\boldsymbol{\Sigma}, \varepsilon}^{\text {triph }}(0, c, 1-c)=\mathcal{F}_{\sigma_{23}, \varepsilon}^{\text {diph }}(c), \quad \forall c \in \mathrm{H}^{1}(\Omega)
$$


which reads, using (1) and (5),

$$
\int_{\Omega} \frac{12}{\varepsilon} F(0, c, 1-c)+\frac{3}{8} \varepsilon\left(\Sigma_{2}+\Sigma_{3}\right)|\nabla c|^{2} \mathrm{~d} x=\int_{\Omega} 12 \frac{\sigma_{23}}{\varepsilon} c^{2}(1-c)^{2}+\frac{3}{4} \sigma_{23} \varepsilon|\nabla c|^{2} \mathrm{~d} x .
$$

Since this equality has to be true for any $c \in \mathrm{H}^{1}(\Omega)$, we can identify the coefficients in front of the capillary terms and the two bulk terms. This gives

$$
\frac{\Sigma_{2}+\Sigma_{3}}{2}=\sigma_{23}, \text { and } F(0, c, 1-c)=\sigma_{23} c^{2}(1-c)^{2}, \forall c \in \mathbb{R}
$$

Hence, changing the role of order parameters, we obtain

$$
\frac{\Sigma_{1}+\Sigma_{2}}{2}=\sigma_{12}, \quad \frac{\Sigma_{1}+\Sigma_{3}}{2}=\sigma_{13}, \quad \frac{\Sigma_{2}+\Sigma_{3}}{2}=\sigma_{23},
$$

and

$$
\left\{\begin{array}{l}
F(c, 1-c, 0)=\sigma_{12} c^{2}(1-c)^{2} \\
F(c, 0,1-c)=\sigma_{13} c^{2}(1-c)^{2} \\
F(0, c, 1-c)=\sigma_{23} c^{2}(1-c)^{2}
\end{array}\right.
$$

It is easily checked that (22) is equivalent to (20).

Let us now look for $F$ under the form

$$
F(\mathbf{C})=\sigma_{12} c_{1}^{2} c_{2}^{2}+\sigma_{13} c_{1}^{2} c_{3}^{2}+\sigma_{23} c_{2}^{2} c_{3}^{2}+P(\mathbf{C})
$$

where $P$ is a smooth function. From (23), we deduce that property (P1) is satisfied if and only if we have

$$
P(c, 1-c, 0)=P(c, 0,1-c)=P(0, c, 1-c)=0, \forall c \in \mathbb{R}
$$

We now use the following straightforward result.

Lemma 3.4. Let $f: \mathbb{R}^{3} \mapsto \mathbb{R}$ be a smooth function. The two following properties are equivalent:

(1) $f(c, 1-c, 0)=f(c, 0,1-c)=f(0, c, 1-c)=0$, for any $c \in \mathbb{R}$.

(2) There exist two smooth functions $g$ and $h$ such that

$$
f\left(c_{1}, c_{2}, c_{3}\right)=c_{1} c_{2} c_{3} g\left(c_{1}, c_{2}, c_{3}\right)+\left(c_{1}+c_{2}+c_{3}-1\right) h\left(c_{1}, c_{2}, c_{3}\right)
$$

From Lemma 3.4 and (25), we deduce that there exist smooth functions $Q$ and $H_{1}$ such that

$$
P(\mathbf{C})=c_{1} c_{2} c_{3} Q(\mathbf{C})+\left(c_{1}+c_{2}+c_{3}-1\right) H_{1}(\mathbf{C}), \quad \forall \mathbf{C} \in \mathbb{R}^{3}
$$

2/ Property (P2) says that the particular triples of the form $(c, 1-c, 0),(c, 0,1-c)$ and $(0, c, 1-c)$ have to be solutions of (8) which implies that

$$
\left(\frac{1}{\Sigma_{j}}\left(\partial_{i} F-\partial_{j} F\right)+\frac{1}{\Sigma_{k}}\left(\partial_{i} F-\partial_{k} F\right)\right)_{\left.\right|_{c_{i}=0, c_{k}=1-c_{j}}}=0, \forall\{i, j, k\}=\{1,2,3\}, \forall c_{j} \in \mathbb{R}
$$


Using (24), we find that relations (27) are equivalent to the following conditions on $P$

$$
\left\{\begin{array}{l}
\left(\frac{1}{\Sigma_{2}}\left(\partial_{1} P-\partial_{2} P\right)+\frac{1}{\Sigma_{3}}\left(\partial_{1} P-\partial_{3} P\right)\right)(0, c, 1-c)=2 \sigma_{23} c(1-c)\left(\frac{1}{\Sigma_{2}}(1-c)+\frac{1}{\Sigma_{3}} c\right), \forall c \in \mathbb{R}, \\
\left(\frac{1}{\Sigma_{1}}\left(\partial_{2} P-\partial_{1} P\right)+\frac{1}{\Sigma_{3}}\left(\partial_{2} P-\partial_{3} P\right)\right)(c, 0,1-c)=2 \sigma_{13} c(1-c)\left(\frac{1}{\Sigma_{1}}(1-c)+\frac{1}{\Sigma_{3}} c\right), \forall c \in \mathbb{R} \\
\left(\frac{1}{\Sigma_{1}}\left(\partial_{3} P-\partial_{1} P\right)+\frac{1}{\Sigma_{2}}\left(\partial_{3} P-\partial_{2} P\right)\right)(c, 1-c, 0)=2 \sigma_{12} c(1-c)\left(\frac{1}{\Sigma_{1}}(1-c)+\frac{1}{\Sigma_{2}} c\right), \forall c \in \mathbb{R} .
\end{array}\right.
$$

Using now (26), it follows

$$
\left\{\begin{array}{l}
\left(\frac{1}{\Sigma_{2}}\left(\partial_{1} P-\partial_{2} P\right)+\frac{1}{\Sigma_{3}}\left(\partial_{1} P-\partial_{3} P\right)\right)(0, c, 1-c)=\left(\frac{1}{\Sigma_{2}}+\frac{1}{\Sigma_{3}}\right) c(1-c) Q(0, c, 1-c), \\
\left(\frac{1}{\Sigma_{1}}\left(\partial_{2} P-\partial_{1} P\right)+\frac{1}{\Sigma_{3}}\left(\partial_{2} P-\partial_{3} P\right)\right)(c, 0,1-c)=\left(\frac{1}{\Sigma_{1}}+\frac{1}{\Sigma_{3}}\right) c(1-c) Q(c, 0,1-c), \\
\left(\frac{1}{\Sigma_{1}}\left(\partial_{3} P-\partial_{1} P\right)+\frac{1}{\Sigma_{2}}\left(\partial_{3} P-\partial_{2} P\right)\right)(c, 1-c, 0)=\left(\frac{1}{\Sigma_{1}}+\frac{1}{\Sigma_{2}}\right) c(1-c) Q(c, 1-c, 0) .
\end{array}\right.
$$

Comparing (28) and (29), we deduce that $Q$ has to fulfill

$$
\left\{\begin{array}{l}
Q(0, c, 1-c)=\Sigma_{2} c+\Sigma_{3}(1-c), \forall c \in \mathbb{R} \\
Q(c, 1-c, 0)=\Sigma_{1} c+\Sigma_{2}(1-c), \forall c \in \mathbb{R} \\
Q(c, 0,1-c)=\Sigma_{1} c+\Sigma_{3}(1-c), \forall c \in \mathbb{R}
\end{array}\right.
$$

We can now look for $Q$ under the form

$$
Q(\mathbf{C})=\Sigma_{1} c_{1}+\Sigma_{2} c_{2}+\Sigma_{3} c_{3}+R(\mathbf{C}), \forall \mathbf{C} \in \mathbb{R}^{3} .
$$

Properties (30) are equivalent to

$$
R(c, 1-c, 0)=R(c, 0,1-c)=R(0, c, 1-c)=0, \quad \forall c \in \mathbb{R},
$$

so that Lemma 3.4 implies that $R$ can be written

$$
R(\mathbf{C})=c_{1} c_{2} c_{3} G(\mathbf{C})+\left(c_{1}+c_{2}+c_{3}-1\right) H_{2}(\mathbf{C}), \forall \mathbf{C} \in \mathbb{R}^{3} .
$$

Gathering together (24), (26), (31) and (32) proves the claim.

Equations (8) only makes sense when the condition (3) is satisfied, that is on the hyperplane $\mathcal{S}$. It is easily seen that, in this context, the term containing the function $H$ in (21) does not play any role in the final system of equations. Hence, from now on, we assume that $H=0$.

\subsection{First examples}

Most of the previous works in the field (see $[13,21]$ ) consider the following definition for the bulk free energy,

$$
F(\mathbf{C})=\widetilde{F}_{0}(\mathbf{C}) \underset{\text { def }}{=} \sigma_{12} c_{1}^{2} c_{2}^{2}+\sigma_{13} c_{1}^{2} c_{3}^{2}+\sigma_{23} c_{2}^{2} c_{3}^{2} .
$$

Theorem 3.2 above says, in particular, that this choice for $F$ is not algebraically consistent with the corresponding diphasic systems as defined above. We show some numerical results in Section 5.3.2 (see Fig. 5) illustrating 
that these non-consistent models are not well-adapted for the study of three component mixtures and may lead to non-physical behaviors.

Let us now concentrate on possible consistent choices for $F$ according to Theorem 3.2. The simplest bulk free energy one can consider to build a consistent model is the following fourth order polynomial

$$
\begin{aligned}
F_{0}(\mathbf{C}) & =\sigma_{12} c_{1}^{2} c_{2}^{2}+\sigma_{13} c_{1}^{2} c_{3}^{2}+\sigma_{23} c_{2}^{2} c_{3}^{2}+c_{1} c_{2} c_{3}\left(\Sigma_{1} c_{1}+\Sigma_{2} c_{2}+\Sigma_{3} c_{3}\right) \\
& =\frac{\Sigma_{1}}{2} c_{1}^{2}\left(c_{2}+c_{3}\right)^{2}+\frac{\Sigma_{2}}{2} c_{2}^{2}\left(c_{1}+c_{3}\right)^{2}+\frac{\Sigma_{3}}{2} c_{3}^{2}\left(c_{1}+c_{2}\right)^{2},
\end{aligned}
$$

the $\left(\Sigma_{i}\right)_{i}$ being defined by $(20)$. When restricted to the hyperplane $\mathcal{S}$ of admissible values of $\mathbf{C}$ we also have the equivalent formula

$$
F_{0}(\mathbf{C})=\frac{\Sigma_{1}}{2} c_{1}^{2}\left(1-c_{1}\right)^{2}+\frac{\Sigma_{2}}{2} c_{2}^{2}\left(1-c_{2}\right)^{2}+\frac{\Sigma_{3}}{2} c_{3}^{2}\left(1-c_{3}\right)^{2}, \quad \forall \mathbf{C} \in \mathcal{S} .
$$

One can see that this function only depends, and in an explicit way, on the physical parameters of the problem $\sigma_{12}, \sigma_{13}$ and $\sigma_{23}$. This is one of the main features of our approach compared to the other models available in the literature for which approximate calibration is needed to determine the parameters of the model (see for instance [13]).

As we have already seen, the model reduces to two coupled Cahn-Hilliard equations. In the particular case $F=F_{0}$, which is often used in numerical simulations, system (8) is equivalent to

$$
\left\{\begin{aligned}
\frac{\partial c_{i}}{\partial t} & =\nabla \cdot\left(\frac{M_{0}}{\Sigma_{i}} \nabla \mu_{i}\right), \quad \text { for } i=1,2 \\
\mu_{i} & =-\frac{3}{4} \varepsilon \Sigma_{i} \Delta c_{i}+\frac{12}{\varepsilon}\left(\Sigma_{i} f\left(c_{i}\right)-\delta c_{1} c_{2}\left(1-c_{1}-c_{2}\right)\right),
\end{aligned}\right.
$$

where $\delta=\frac{6 \Sigma_{1} \Sigma_{2} \Sigma_{3}}{\Sigma_{1} \Sigma_{2}+\Sigma_{1} \Sigma_{3}+\Sigma_{2} \Sigma_{3}}$ and $f(x)=x(1-x)(1-2 x)$. The concentration of the third component is then given by $c_{3}=1-c_{1}-c_{2}$ and the chemical potential $\mu_{3}$ by (9). As we have already seen, any of the three concentrations $c_{i}$ can be eliminated by this procedure which leads to fully equivalent systems.

The simple system (36) above will be used in many situations (see Sect. 5). Nevertheless, this model suffers from some drawbacks in particular cases:

- In the total spreading case, that is when one of the $\Sigma_{i}$ is negative, assumption (13) is never satisfied. Indeed, if for instance $\Sigma_{1}$ is negative, it is easily seen that

$$
F_{0}(-1,1,1)=2 \Sigma_{1}<0
$$

Of course the triple $(-1,1,1)$ is not physically meaningful, but we have already seen that the nondegenerate Cahn-Hilliard system with smooth bulk free energies does not ensure that the concentrations remain non-negative (see Rem. 1.1).

In fact, it may happen that, even under condition (11), $F_{0}$ is not bounded from below on the hyperplane $\mathcal{S}$ (take for instance $\Sigma_{1}=\Sigma_{2}=1$ and $\Sigma_{3}=-0.2$ ). In that case, Theorem 2.4 does not apply and in fact, we guess that the system (8) is ill-posed, which is confirmed by numerical simulations which blow up in very short times.

- Even though the system (8) obtained with $F=F_{0}$ is algebraically consistent with diphasic systems and well-posed at least when all the $\Sigma_{i}$ are positive, it will appear in Section 3.4 that this system is not always dynamically consistent with diphasic systems. This means that, even if diphasic solutions of (8) (i.e. triples of the form $(c, 1-c, 0))$ do exist, these solutions may not remain "diphasic" when we apply small perturbations of the initial data. This phenomenon may produce non physical instabilities of the interfaces (see the numerical simulations in Sect. 5 and in particular Fig. 6). 
That is the reason why we study in the sequel a more general possible choice for the bulk free energy $F$ which will ensure well-posedness, but also algebraic and dynamical consistency of the model.

\subsection{Higher order bulk free energy}

As we have seen before, if one of coefficients $\Sigma_{i}$ is negative, and even in some cases where all the $\Sigma_{i}$ are positive, it can be necessary to consider more complicated bulk free energies allowed by Theorem 3.2 to preserve the algebraic consistency with binary systems. We will concentrate in the sequel on the following particular case

$$
F_{\Lambda, \alpha}(\mathbf{C})=F_{0}(\mathbf{C})+\Lambda c_{1}^{2} c_{2}^{2} c_{3}^{2}\left(\varphi_{\alpha}\left(c_{1}\right)+\varphi_{\alpha}\left(c_{2}\right)+\varphi_{\alpha}\left(c_{3}\right)\right)
$$

where $\Lambda$ is a non-negative real parameter and $\varphi_{\alpha}$ is the function $\varphi_{\alpha}: x \mapsto \frac{1}{\left(1+x^{2}\right)^{\alpha}}, \alpha \geq 0$. When $\Lambda=0$, we recover the bulk free energy $F_{0}$ introduced in the previous paragraph. The simple case $\alpha=0$ leads to the following sixth order polynomial bulk free energy

$$
F_{\Lambda, 0}(\mathbf{C})=F_{0}(\mathbf{C})+3 \Lambda c_{1}^{2} c_{2}^{2} c_{3}^{2}
$$

which will be used in the numerical simulations presented in Section 5. Unfortunately, our analysis does not apply in 3D for this limit case $\alpha=0$. That is the reason why we consider here (37) instead of (38).

Let us introduce $\Phi_{\alpha}(x)=x^{2} \varphi_{\alpha}(x)$. We state here, for further use, the main useful properties of $\varphi_{\alpha}$ and $\Phi_{\alpha}$.

Lemma 3.5. (1) For any $\alpha \geq 0$, there exists $K_{\alpha}>0$ such that

(2) We have

$$
\begin{aligned}
& \varphi_{\alpha}(x) \leq \frac{K_{\alpha}}{|x|^{2 \alpha}}, \quad\left|\varphi_{\alpha}^{\prime}(x)\right| \leq \frac{K_{\alpha}}{|x|^{2 \alpha+1}}, \quad \forall x \in \mathbb{R}, \\
& \left|x-\frac{1}{2} \Phi_{\alpha}^{\prime}(x)\right| \leq K_{\alpha} \frac{|x|^{3}}{1+x^{2}} \leq \frac{K_{\alpha}}{2}|x|^{2}, \quad \forall x \in \mathbb{R} .
\end{aligned}
$$

$$
\begin{gathered}
\Phi_{\alpha} \text { is convex, for any } \alpha \in\left[0, \frac{8}{17}\right], \\
x \Phi_{\alpha}^{\prime}(x) \geq 0, \quad \forall x \in \mathbb{R}, \text { for any } \alpha \in[0,1] .
\end{gathered}
$$

\subsubsection{Well-posedness of the Cahn-Hilliard system}

We want to show in this section that the system (8) is well-posed when the bulk free energy is defined by (37). To this end, it is enough to apply Theorem 2.4. The main point here, is to prove that $F_{\Lambda, \alpha}$ satisfies the assumptions of this theorem, in particular in the 3D case. From now on, we make the following assumption

$$
\left\{\begin{array}{l}
\alpha \in[0,1], \text { if } d=2, \\
\left.\alpha \in] 0, \frac{8}{17}\right], \text { if } d=3
\end{array}\right.
$$

(1) Polynomial growth:

Using Lemma 3.5, it is straightforward to check that for any non-negative $\Lambda$ and $\alpha$, there exists $B_{1}, B_{2}>0$ such that

$$
\begin{aligned}
\left|F_{\Lambda, \alpha}(\mathbf{C})\right| & \leq B_{1}|\mathbf{C}|^{6-2 \alpha}+B_{2}, \quad \forall \mathbf{C} \in \mathcal{S}, \\
\left|D F_{\Lambda, \alpha}(\mathbf{C})\right| & \leq B_{1}|\mathbf{C}|^{5-2 \alpha}+B_{2}, \quad \forall \mathbf{C} \in \mathcal{S}, \\
\left|D^{2} F_{\Lambda, \alpha}(\mathbf{C})\right| & \leq B_{1}|\mathbf{C}|^{4}+B_{2}, \quad \forall \mathbf{C} \in \mathcal{S} .
\end{aligned}
$$

Therefore, assumptions (14)-(16) are fulfilled, for $d=2$ and $d=3$. Notice that the exponent 4 in the right-hand side of (43) is sharp (see (44) below). 
(2) Bound from below for the Hessian:

One easily checks, using (43), that assumption (17) is satisfied, with $q=4$, by $F_{\Lambda, \alpha}$ for any $\Lambda \geq 0$ and any $\alpha \geq 0$. Unfortunately, in the 3D case, we absolutely need that $q<4$.

Of course all the terms in $F_{0}$ are fourth-order polynomial terms and contribute to $D^{2} F_{\Lambda, \alpha}$ only with second order terms. Let us now consider one of the additional terms in $F_{\Lambda, \alpha}$, say for instance $G(\mathbf{C})=\Lambda c_{1}^{2} c_{2}^{2} c_{3}^{2} \varphi_{\alpha}\left(c_{1}\right)=\Lambda \Phi_{\alpha}\left(c_{1}\right) c_{2}^{2} c_{3}^{2}$. Using Lemma 3.5 one can easily see that

$$
\left|\partial_{i} \partial_{j} G(\mathbf{C})\right| \leq K|\mathbf{C}|^{4-2 \alpha},
$$

as soon as $(i, j) \neq(1,1)$. Hence, these terms contribute to the inequality (17) with a power $q=4-2 \alpha<4$ thanks to assumption (40) for $d=3$. It remains to consider the term

$$
\partial_{1}^{2} G(\mathbf{C})=\Phi_{\alpha}^{\prime \prime}\left(c_{1}\right) c_{2}^{2} c_{3}^{2},
$$

which is of order 4 and not below. Nevertheless, since $\alpha$ satisfies (40) we know from Lemma 3.5 that $\Phi_{\alpha}$ is convex and in particular, the term $\partial_{1}^{2} G(\mathbf{C})$ above is non-negative. As a consequence its contribution to $\left(D^{2} F_{\Lambda, \alpha} \cdot \xi, \xi\right)$ is $\partial_{1}^{2} G\left|\xi_{1}\right|^{2}$ which is non-negative. This proves that the bound from below (17) is fulfilled by $F_{\Lambda, \alpha}$ with $q=4-2 \alpha$.

(3) Bound from below:

First of all, it is clear from (35) and (37) that $F_{\Lambda, \alpha}$ is non-negative on $\mathcal{S}$ for any $\Lambda \geq 0$ and any $\alpha \geq 0$ as soon as all the coefficients $\left(\Sigma_{i}\right)_{i}$ are positive. In the case where one of the $\left(\Sigma_{i}\right)_{i}$ is negative we show below that $F_{\Lambda, \alpha}$ is bounded from below as soon as $\Lambda>0$.

In particular, using Theorem 2.4, this proves that system (8) with $F=F_{\Lambda, \alpha}$ is well posed for any values of $\left(\Sigma_{i}\right)$ satisfying (11)-(12) as soon as $\Lambda>0$ and (40) holds. Furthermore, (45) shows that the additional higher order term in the bulk free energy acts as a stabilizing term which tends to the ideal case where the bulk free energy is non-negative as $\Lambda$ tends to infinity.

Proposition 3.6. Let $\sigma_{12}, \sigma_{13}$ and $\sigma_{23}$ be three positive numbers and $\Sigma_{1}, \Sigma_{2}$ and $\Sigma_{3}$ defined by (20). For any $\alpha$ satisfying (40) and any $\Lambda>0$ the bulk free energy $F_{\Lambda, \alpha}$ defined by (34) and (37) is bounded from below on the hyperplane $\mathcal{S}$ and we have

$$
\lim _{\Lambda \rightarrow+\infty}\left(\inf _{\mathcal{S}} F_{\Lambda, \alpha}\right)=0 .
$$

The proof of this result is given in Appendix A.

(4) Non-negativity:

More interestingly, we are going to show that under suitable conditions and for $\Lambda$ large enough, the bulk free energy $F_{\Lambda, \alpha}$ is non-negative and have only the triples $(1,0,0),(0,1,0)$ and $(0,0,1)$ as minimizing states on $\mathcal{S}$.

Proposition 3.7. Let $\alpha$ satisfying (40). There exists $\Lambda_{0}>0$ such that $\inf _{\mathcal{S}} F_{\Lambda, \alpha}=0$ for all $\Lambda \geq \Lambda_{0}$ if and only if we have

$$
\Sigma_{1} \Sigma_{2}+\Sigma_{1} \Sigma_{3}+\Sigma_{2} \Sigma_{3}>0
$$

The proof of this result is given in Appendix A.

Notice that we find here the condition (46) which is exactly the same as (11).

\subsubsection{Conclusions}

We sum up here the conclusions of the previous study:

- If all the $\left(\Sigma_{i}\right)_{i}$ defined by (20) are positive then, problem (8) with $F=F_{\Lambda, \alpha}$ is well-posed for any $\Lambda \geq 0$ and any $\alpha$ satisfying (40). In particular the simplest choice $F=F_{0}$ is always acceptable. Furthermore, the three pure components states $(1,0,0),(0,1,0)$ and $(0,0,1)$ are the unique minimizers of the energy. 


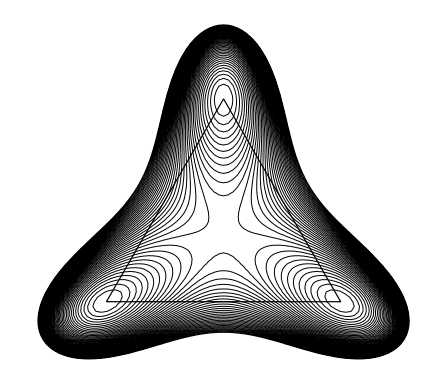

$\Sigma_{1}=\Sigma_{2}=\Sigma_{3}=4, F=\tilde{F}_{0}$

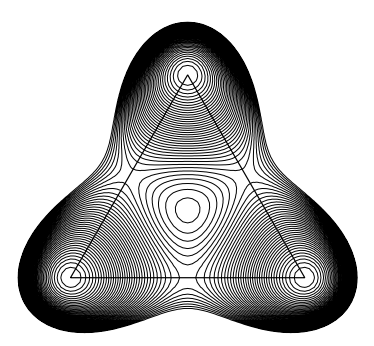

$\Sigma_{1}=\Sigma_{2}=\Sigma_{3}=4, F=F_{0}$

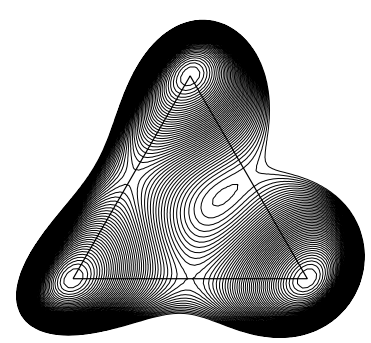

$\Sigma_{1}=6, \Sigma_{2}=8, \Sigma_{3}=4, F=F_{0}$

FiguRE 1. Fourth order polynomial bulk free energies.

- If one of the $\left(\Sigma_{i}\right)_{i}$ is negative, then $F_{0}$ may be unbounded from below. Nevertheless, under condition (46), for any $\Lambda>0$ and any $\alpha$ satisfying (40), the problem (8) with $F=F_{\Lambda, \alpha}$ is well-posed.

Furthermore, always under the condition (46), for any $\alpha$ satisfying (40) and any $\Lambda>0$ large enough, the bulk free energy $F_{\Lambda, \alpha}$ is non-negative and has only the expected three minimizers.

We can observe the differences between different bulk free energies by looking at the isolines of the function $F$ in barycentric coordinates. More precisely we draw an equilateral triangle (the so-called Gibbs triangle) whose vertices represent the three pure phases $(1,0,0),(0,1,0)$ and $(0,0,1)$. Then, we can look at any $\mathbf{C} \in \mathcal{S}$ as the barycentric coordinates of any point of the plane with respect to the three vertices of the triangle. The points located at the interior of the Gibbs triangle represents physically admissible values of the concentrations. Recall that the Cahn-Hilliard equation will possibly lead to values of $\mathbf{C}$ outside this triangle (at least when the mobility is non-generate see Rem. 1.1).

In Figure 1, we compare first in the case of three identical surface tensions (left and center plots), the difference between the non-consistent $\tilde{F}_{0}$ and the consistent $F_{0}$ bulk free energy. In the non-consistent case the center of the Gibbs triangle is a kind of saddle-point for $F$ whereas in the consistent case, the center is a local maximum of $F$. Furthermore, we see that the least energy path between two vertices of the Gibbs triangle (that is between two pure phase states) is exactly the corresponding edge of the triangle for each consistent bulk free energy, which is not the case for non-consistent ones. Actually, this is a geometrical characterization of the algebraic consistency property. Since the evolution of the system is driven by the minimization of the total energy we understand easily that the choice of $\tilde{F}_{0}$ will lead to non-physical apparition of one phase in the interface between the other two. In the right plot we show that the shape of $F_{0}$ has the same structure (with a local maximum point inside the Gibbs triangle) even for three different surface tensions.

In Figure 2, we concentrate on a total spreading case, and we compare the plots obtained for $F_{\Lambda, 0}$ for various values of $\Lambda$. For very small values of $\Lambda$ (left plot) the function $F_{\Lambda, 0}$ takes negative values (the light gray zone at the top-right corner of the plot) and the system will not behave correctly since it will try to reach this global negative minimum for the energy. If we increase $\Lambda$ (center plot) we find that $F_{\Lambda, 0}$ is non-negative but has many local minima that the system can try to reach in order to minimize the energy. We are in a case where the model will not be dynamically consistent (see Sect. 3.4 below) and instabilities will appear. Finally, for large enough $\Lambda$ (right plot), $F_{\Lambda, 0}$ is of course non-negative, has no other local minima than the three vertices of the Gibbs triangle and possess a local maximum inside the Gibbs triangle. Moreover, just like in Figure 1, the three edges of the Gibbs triangle appear to be the least energy paths between its vertices. Hence, for such a value of $\Lambda$ we recover the same shape as in the center and right plots of Figure 1. 


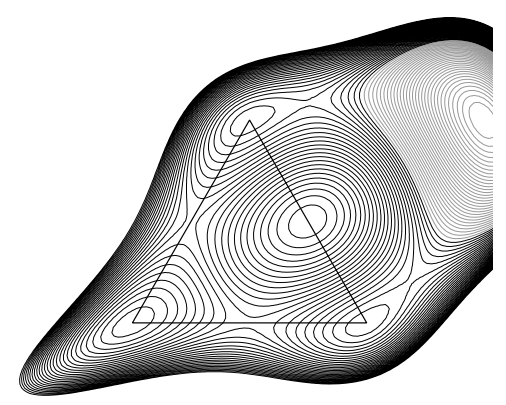

$\Lambda=0.4$

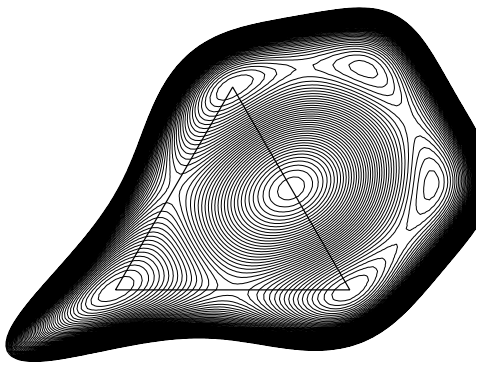

$\Lambda=0.7$

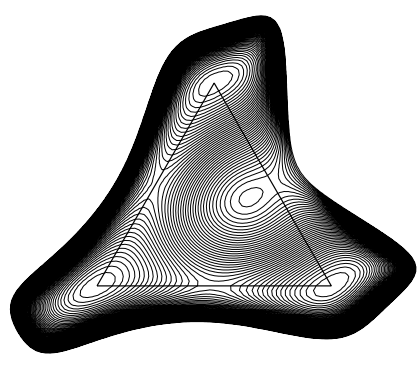

$\Lambda=10.0$

FigurE 2. Total spreading case $\Sigma_{1}=\Sigma_{2}=4, \Sigma_{3}=-0.8$. Various values of $\Lambda$.

\subsection{Dynamical consistency of the models}

Our model is built to ensure algebraic consistency with diphasic mixtures. Hence for any $\Lambda \geq 0$, we have particular exact solutions of $(8)$ of the form $(c, 1-c, 0)$ where $c$ satisfies a "diphasic" Cahn-Hilliard equation as shown in (2). We will now prove that our models are also dynamically consistent in the sense that such particular "diphasic" solutions are stable under perturbations of initial data. This point is crucial in view of numerical computations since it ensures that numerical errors near those particular initial data will decrease exponentially in time. Furthermore, in practical fully ternary situations, dynamically consistent models lead to much more stable interfaces between two of the components as illustrated in Section 5.3.3.

For any $f \in L^{1}(\Omega)$, we define its mean-value over $\Omega, m(f)=\frac{1}{|\Omega|} \int_{\Omega} f(x) \mathrm{d} x$. Let us introduce the spaces $L_{m}^{2}(\Omega)$ (resp. $\left.H_{m}^{1}(\Omega)\right)$ to be the sets of functions $f$ in $L^{2}(\Omega)$ (resp. $H^{1}(\Omega)$ ) such that $m(f)=0$. Thanks to the Poincaré inequality, $c \mapsto|\nabla c|_{L^{2}}$ is a norm on $H_{m}^{1}(\Omega)$ that we denote by $|\cdot|_{1}$. Furthermore, we recall that for any $f \in L_{m}^{2}(\Omega)$ there exists a unique $u \in H_{m}^{1}(\Omega)$ such that

$$
\left\{\begin{aligned}
-\Delta u & =f, \text { in } \Omega \\
\frac{\partial u}{\partial n} & =0, \text { on } \partial \Omega .
\end{aligned}\right.
$$

We denote this unique element $u$ by $u=(-\Delta)^{-1} f$ and we note $|f|_{-1}=\left|(-\Delta)^{-1} f\right|_{1}=\left((-\Delta)^{-1} f, f\right)_{\mathrm{L}^{2}}^{\frac{1}{2}}$ which is a norm on $L_{m}^{2}(\Omega)$. We recall finally that we have the interpolation property

$$
|f|_{\mathrm{L}^{2}} \leq|f|_{-1}^{\frac{1}{2}}|f|_{1}^{\frac{1}{2}}, \quad \forall f \in H_{m}^{1}(\Omega)
$$

Theorem 3.8. Let $\alpha$ satisfy (40). Assume that conditions (11)-(12) and assumptions (13)-(17) hold. There exists $\Lambda_{1}>0$ such that for any $\Lambda \geq \Lambda_{1}$, diphasic solutions of system (8) are stable in the following sense:

For any $\mathcal{K}>0$, there exists $\delta, \gamma>0$ such that for any $\mathbf{C}^{0} \in \mathcal{S}$ such that $\left|\mathbf{C}^{0}\right|_{\mathrm{H}^{1}} \leq \mathcal{K}$ :

$$
\left|c_{j}^{0}\right|_{-1} \leq \sqrt{\delta} \text { and } \int_{\Omega} c_{j}^{0} \mathrm{~d} x=0 \Longrightarrow\left|c_{j}(t)\right|_{-1} \leq\left|c_{j}^{0}\right|_{-1} \mathrm{e}^{-\gamma t}, \forall t>0
$$

As a consequence, this theorem and (47) imply that if $c_{j}^{0}$ is small enough and with zero mean then $c_{j}(t)$ tends to zero exponentially in time in all the spaces $H^{s}(\Omega)$ with $s<1$. 
Theorem 3.9. If $\boldsymbol{\Sigma}$ satisfy

$$
\Sigma_{i}>\frac{\Sigma_{T}}{2}>0, \quad \forall i=\{1,2,3\}
$$

the claim of Theorem 3.8 holds with $\Lambda=0$.

This last result means that, under assumption (48), the simplest possible algebraically consistent model, that is the one for $F=F_{0}$, is well-posed and dynamically consistent with diphasic systems. In Section 5.3.3, we give numerical results showing that, when the condition (48) is not satisfied, the model can present non physical instabilities.

We give now the proof of Theorem 3.8 for $d=3$, the bidimensional case being easier.

Proof. Assume for instance that $j=1$. Since $m\left(c_{1}^{0}\right)=0$ we know that $m\left(c_{1}(t)\right)=0$ for any $t \geq 0$. The equation satisfied by $c_{1}$ reads

$$
\frac{\partial c_{1}}{\partial t}=\frac{M_{0}}{\Sigma_{1}} \Delta \mu_{1}
$$

Applying the operator $(-\Delta)^{-1}$ defined above to this equation gives

$$
\frac{\partial(-\Delta)^{-1} c_{1}}{\partial t}=-\frac{M_{0}}{\Sigma_{1}}\left(\mu_{1}-m\left(\mu_{1}\right)\right)
$$

so that, taking the $\mathrm{L}^{2}(\Omega)$ inner product of this equation with $c_{1}(t)$ which has a zero mean-value, we get

$$
\frac{1}{2} \frac{\mathrm{d}}{\mathrm{d} t}\left((-\Delta)^{-1} c_{1}, c_{1}\right)_{\mathrm{L}^{2}}=-\frac{M_{0}}{\Sigma_{1}} \int_{\Omega}\left(\mu_{1}-m\left(\mu_{1}\right)\right) c_{1} \mathrm{~d} x=-\frac{M_{0}}{\Sigma_{1}} \int_{\Omega} \mu_{1} c_{1} \mathrm{~d} x .
$$

Using now the definition of $\mu_{1}$ and of $F_{\Lambda, \alpha}$ we get

$$
\begin{aligned}
\frac{1}{2} \frac{\mathrm{d}}{\mathrm{d} t}\left((-\Delta)^{-1} c_{1}, c_{1}\right)_{\mathrm{L}^{2}}+ & \frac{3 M_{0} \varepsilon}{4}\left|\nabla c_{1}\right|_{\mathrm{L}^{2}}^{2} \\
& +\frac{12 M_{0}}{\varepsilon} \int_{\Omega} c_{1}^{2}\left[1-\beta_{1} c_{2} c_{3}+2 \Lambda \gamma_{1} c_{2}^{2} c_{3}^{2}\left(\varphi_{\alpha}\left(c_{2}\right)+\varphi_{\alpha}\left(c_{3}\right)\right)\right]+\Lambda \gamma_{1} c_{1} \Phi_{\alpha}^{\prime}\left(c_{1}\right) c_{2}^{2} c_{3}^{2} \mathrm{~d} x \\
= & \frac{12 M_{0}}{\varepsilon} \int_{\Omega} c_{1}^{3}\left(3-2 c_{1}\right) \mathrm{d} x \\
& +\frac{24 M_{0} \Lambda}{\varepsilon} \frac{\Sigma_{T}}{3 \Sigma_{1} \Sigma_{2} \Sigma_{3}} \int_{\Omega} c_{1}^{3} c_{2} c_{3}\left(\Sigma_{2} c_{2}+\Sigma_{3} c_{3}\right)\left(\varphi_{\alpha}\left(c_{1}\right)+\varphi_{\alpha}\left(c_{2}\right)+\varphi_{\alpha}\left(c_{3}\right)\right) \mathrm{d} x \\
& +\frac{12 M_{0} \Lambda}{\varepsilon} \frac{\Sigma_{T}}{3 \Sigma_{1} \Sigma_{2} \Sigma_{3}} \int_{\Omega} c_{1}^{3} c_{2}^{2} c_{3}^{2}\left(\Sigma_{3} \varphi_{\alpha}^{\prime}\left(c_{2}\right)+\Sigma_{2} \varphi_{\alpha}^{\prime}\left(c_{3}\right)\right) \mathrm{d} x
\end{aligned}
$$

where

$$
\beta_{1}=\frac{6 \Sigma_{2} \Sigma_{3}}{\Sigma_{1} \Sigma_{2}+\Sigma_{1} \Sigma_{3}+\Sigma_{2} \Sigma_{3}}=\frac{2 \Sigma_{T}}{\Sigma_{1}} \quad \text { and } \quad \gamma_{1}=\frac{\Sigma_{T}}{3 \Sigma_{1}}\left(\frac{1}{\Sigma_{2}}+\frac{1}{\Sigma_{3}}\right)=\frac{\left(\Sigma_{2}+\Sigma_{3}\right)}{\Sigma_{1} \Sigma_{2}+\Sigma_{1} \Sigma_{3}+\Sigma_{2} \Sigma_{3}} \text {. }
$$

Notice that since conditions (11) and (12) hold we have $\gamma_{1}>0$. Then, in the left-hand side of the above equality the term $\int_{\Omega} 2 \Lambda \gamma_{1} c_{1}^{2} c_{2}^{2} c_{3}^{2}\left(\varphi_{\alpha}\left(c_{2}\right)+\varphi_{\alpha}\left(c_{3}\right)\right) \mathrm{d} x$ is non-negative. On the left-hand side, there remains one integral term that we write:

$$
\begin{aligned}
\int_{\Omega} c_{1}^{2}\left(1-\beta_{1} c_{2} c_{3}\right)+\Lambda \gamma_{1} c_{1} \Phi_{\alpha}^{\prime}\left(c_{1}\right) c_{2}^{2} c_{3}^{2} \mathrm{~d} x=\frac{1}{2} \int_{\Omega} c_{1} \Phi_{\alpha}^{\prime}\left(c_{1}\right)\left(1-\beta_{1} c_{2} c_{3}+2 \Lambda \gamma_{1} c_{2}^{2} c_{3}^{2}\right) \mathrm{d} x & +\int_{\Omega} c_{1}\left(c_{1}-\frac{1}{2} \Phi_{\alpha}^{\prime}\left(c_{1}\right)\right)\left(1-\beta_{1} c_{2} c_{3}\right) \mathrm{d} x
\end{aligned}
$$


We remark that if $\Lambda>\frac{\beta_{1}^{2}}{8 \gamma_{1}}>0$, then $1-\beta_{1} X+2 \Lambda \gamma_{1} X^{2} \geq 1-\frac{\beta_{1}^{2}}{8 \Lambda \gamma_{1}}>0$, for all $X \in \mathbb{R}$. Hence, in (50) we have

$$
\frac{1}{2} \int_{\Omega} c_{1} \Phi_{\alpha}^{\prime}\left(c_{1}\right)\left(1-\beta_{1} c_{2} c_{3}+2 \Lambda \gamma_{1} c_{2}^{2} c_{3}^{2}\right) \mathrm{d} x>\frac{1}{2} \int_{\Omega} c_{1} \Phi_{\alpha}^{\prime}\left(c_{1}\right)\left(1-\frac{\beta_{1}^{2}}{8 \Lambda \gamma_{1}}\right) \mathrm{d} x \geq 0 .
$$

Consequently, we obtain

$$
\begin{aligned}
\frac{1}{2} \frac{\mathrm{d}}{\mathrm{d} t}\left((-\Delta)^{-1} c_{1}, c_{1}\right)_{\mathrm{L}^{2}} & +\frac{3 M_{0} \varepsilon}{4}\left|\nabla c_{1}\right|_{\mathrm{L}^{2}}^{2}+\frac{K_{0}}{\varepsilon} \int_{\Omega} c_{1} \Phi_{\alpha}^{\prime}\left(c_{1}\right) \mathrm{d} x \\
\leq & \frac{12 M_{0}}{\varepsilon} \int_{\Omega} c_{1}^{3}\left(3-2 c_{1}\right) \mathrm{d} x-\frac{12 M_{0}}{\varepsilon} \int_{\Omega} c_{1}\left(c_{1}-\frac{1}{2} \Phi_{\alpha}^{\prime}\left(c_{1}\right)\right)\left(1-\beta_{1} c_{2} c_{3}\right) \mathrm{d} x \\
+ & \frac{24 M_{0} \Lambda}{\varepsilon} \frac{\Sigma_{T}}{3 \Sigma_{1} \Sigma_{2} \Sigma_{3}} \int_{\Omega} c_{1}^{3} c_{2} c_{3}\left(\Sigma_{2} c_{2}+\Sigma_{3} c_{3}\right)\left(\varphi_{\alpha}\left(c_{1}\right)+\varphi_{\alpha}\left(c_{2}\right)+\varphi_{\alpha}\left(c_{3}\right)\right) \mathrm{d} x \\
& +\frac{12 M_{0} \Lambda}{\varepsilon} \frac{\Sigma_{T}}{3 \Sigma_{1} \Sigma_{2} \Sigma_{3}} \int_{\Omega} c_{1}^{3} c_{2}^{2} c_{3}^{2}\left(\Sigma_{3} \varphi_{\alpha}^{\prime}\left(c_{2}\right)+\Sigma_{2} \varphi_{\alpha}^{\prime}\left(c_{3}\right)\right) \mathrm{d} x .
\end{aligned}
$$

Let $I_{1}, \ldots, I_{4}$ be the four integrals in the right-hand side above. For the term $I_{1}$, we have the estimate

$$
\left|I_{1}\right| \leq K\left|c_{1}\right|_{\mathrm{L}^{3}}^{3}+K\left|c_{1}\right|_{\mathrm{L}^{4}}^{4} .
$$

Thanks to Lemma 3.5, we get

$$
\begin{gathered}
\left|I_{2}\right| \leq K_{\alpha} \int_{\Omega}\left|c_{1}\right|^{3}\left(1+\left|c_{2}\right|\left|c_{3}\right|\right) \mathrm{d} x \leq K_{\alpha}\left|c_{1}\right|_{\mathrm{L}^{3}}^{3}+K_{\alpha}\left|c_{1}\right|_{\mathrm{L}^{\frac{9}{2}}}^{3}|\mathbf{C}|_{\mathrm{L}^{6}}^{2} \\
\left|I_{3}\right| \leq K_{\alpha} \int_{\Omega}\left|c_{1}\right|^{3-2 \alpha}|\mathbf{C}|^{3}+\left|c_{1}\right|^{3}|\mathbf{C}|^{3-2 \alpha} \mathrm{d} x \\
\leq K_{\alpha}\left|c_{1}\right|_{\mathrm{L}^{6-4 \alpha}}^{3-2 \alpha}|\mathbf{C}|_{\mathrm{L}^{6}}^{3}+K_{\alpha}\left|c_{1}\right|_{\mathrm{L}^{\frac{18}{3+2 \alpha}}}^{3}|\mathbf{C}|_{\mathrm{L}^{6}}^{3-2 \alpha}
\end{gathered}
$$

and in the same way

$$
\left|I_{4}\right| \leq K_{\alpha} \int_{\Omega}\left|c_{1}\right|^{3}|\mathbf{C}|^{3-2 \alpha} \mathrm{d} x \leq K_{\alpha}\left|c_{1}\right|_{\mathrm{L}^{\frac{18}{3+2 \alpha}}}^{3}|\mathbf{C}|_{\mathrm{L}^{6}}^{3-2 \alpha} .
$$

Applying Theorem 2.4, the norm of the solution $\mathbf{C}$ in $\mathrm{L}^{\infty}\left(\mathbb{R}^{+}, \mathrm{H}^{1}\right)$ depends only on $\mathcal{K}$. As we assumed that $0<\alpha \leq \frac{8}{17}$, using the Sobolev embedding $\mathrm{H}^{1}(\Omega) \subset \mathrm{L}^{6}(\Omega)$ and Poincaré's inequality, we get

$$
\frac{1}{2} \frac{\mathrm{d}}{\mathrm{d} t}\left((-\Delta)^{-1} c_{1}, c_{1}\right)_{\mathrm{L}^{2}}+\frac{\varepsilon}{K_{p}}\left|c_{1}\right|_{\mathrm{H}^{1}}^{2} \leq K_{\alpha}\left|c_{1}\right|_{\mathrm{L}^{\frac{9}{2}}}^{3}+K\left|c_{1}\right|_{\mathrm{L}^{3}}^{3}+K\left|c_{1}\right|_{\mathrm{L}^{4}}^{4}+K_{\alpha}\left|c_{1}\right|_{\mathrm{L}^{\frac{18}{3+2 \alpha}}}^{3}+K_{\alpha}\left|c_{1}\right|_{\mathrm{L}^{6-4 \alpha}}^{3-2 \alpha} .
$$

From the embeddings $\mathrm{H}^{\frac{5}{6}} \subset \mathrm{L}^{\frac{9}{2}} \subset \mathrm{L}^{4} \subset \mathrm{L}^{3}$ and the interpolation $\mathrm{H}^{\frac{5}{6}}=\left[\mathrm{H}^{1}, \mathrm{~L}^{2}\right]_{\frac{1}{6}}$, it follows

$$
K_{\alpha}\left|c_{1}\right|_{\mathrm{L}^{\frac{9}{2}}}^{3}+K\left|c_{1}\right|_{\mathrm{L}^{3}}^{3}+K\left|c_{1}\right|_{\mathrm{L}^{4}}^{4} \leq K_{\alpha}\left|c_{1}\right|_{\mathrm{L}^{2}}^{\frac{1}{2}}\left|c_{1}\right|_{\mathrm{H}^{1}}^{\frac{5}{2}}+K_{\alpha}\left|c_{1}\right|_{\mathrm{L}^{2}}^{\frac{2}{3}}\left|c_{1}\right|_{\mathrm{H}^{1}}^{\frac{10}{3}}
$$

In the same way, from $\mathrm{H}^{\frac{3-3 \alpha}{3-2 \alpha}} \subset \mathrm{L}^{6-4 \alpha}, \mathrm{H}^{\frac{3-3 \alpha}{3-2 \alpha}}=\left[\mathrm{H}^{1}, \mathrm{~L}^{2}\right]_{\frac{\alpha}{3-2 \alpha}}$ and the Poincaré inequality, one gets

$$
\left|c_{1}\right|_{\mathrm{L}^{6-4 \alpha}}^{3-2 \alpha} \leq K_{\alpha}\left|c_{1}\right|_{\mathrm{L}^{2}}^{\alpha}\left|c_{1}\right|_{\mathrm{H}^{1}}^{3-3 \alpha} \leq K_{\alpha}\left|c_{1}\right|_{\mathrm{L}^{2}}^{\frac{\alpha}{4}}\left|c_{1}\right|_{\mathrm{H}^{1}}^{3-\frac{9}{4} \alpha},
$$

and finally using $\mathrm{H}^{\frac{3-\alpha}{3}} \subset \mathrm{L}^{\frac{18}{3+2 \alpha}}$ and $\mathrm{H}^{\frac{3-\alpha}{3}}=\left[\mathrm{H}^{1}, \mathrm{~L}^{2}\right]_{\frac{\alpha}{3}}$, one obtains

$$
\left|c_{1}\right|_{\mathrm{L}^{\frac{18}{3+2 \alpha}}}^{3+2 \alpha} \leq K_{\alpha}\left|c_{1}\right|_{\mathrm{L}^{2}}^{\alpha}\left|c_{1}\right|_{\mathrm{H}^{1}}^{3-\alpha} .
$$


Introducing now $y(t)=\left((-\Delta)^{-1} c_{1}, c_{1}\right)_{\mathrm{L}^{2}}$ and $z(t)=\left|c_{1}\right|_{\mathrm{H}^{1}}^{2}$ and using (47), we obtain from (51) and the above estimates

$$
\frac{1}{2} \frac{\mathrm{d}}{\mathrm{d} t} y(t)+\frac{\varepsilon}{K_{p}} z(t) \leq K_{\alpha}\left(z^{\frac{11}{8}} y^{\frac{1}{8}}+z^{\frac{11}{6}} y^{\frac{1}{6}}+z^{\frac{24-17 \alpha}{16}} y^{\frac{\alpha}{16}}+z^{\frac{6-\alpha}{4}} y^{\frac{\alpha}{4}}\right), \quad \forall t \geq 0 .
$$

From Theorem 2.4, we know that $\mathrm{z}$ is uniformly bounded in time by a constant $C_{\mathcal{K}}$ which depends only on $\mathcal{K}$ and that $y \in \mathcal{C}^{0}(\mathbb{R})$. Let us now choose $\delta$ small enough so that

$$
K_{\alpha}\left(C_{\mathcal{K}}^{\frac{3}{8}} \delta^{\frac{1}{8}}+C_{\mathcal{K}}^{\frac{5}{6}} \delta^{\frac{1}{6}}+C_{\mathcal{K}}^{\frac{8-17 \alpha}{16}} \delta^{\frac{\alpha}{16}}+C_{\mathcal{K}}^{\frac{2-\alpha}{4}} \delta^{\frac{\alpha}{4}}\right) \leq \frac{\varepsilon}{4 K_{p}} .
$$

Notice that $8-17 \alpha \geq 0$ thanks to (40). Since by assumption we have $y(0) \leq \delta$, then the continuity of $y$ and $z$ with respect to the time imply that there exists a maximal time $\left.\left.T_{0} \in\right] 0 ;+\infty\right]$ such that

$$
K_{\alpha}\left(z^{\frac{3}{8}} y^{\frac{1}{8}}+z^{\frac{5}{6}} y^{\frac{1}{6}}+z^{\frac{8-17 \alpha}{16}} y^{\frac{\alpha}{16}}+z^{\frac{2-\alpha}{4}} y^{\frac{\alpha}{4}}\right) \leq \frac{\varepsilon}{2 K_{p}}, \quad \forall 0 \leq t<T_{0} .
$$

Hence, (52) leads to

and therefore

$$
\frac{1}{2} \frac{\mathrm{d}}{\mathrm{d} t} y(t)+\frac{\varepsilon}{K_{P}} z(t) \leq \frac{\varepsilon}{2 K_{p}} z(t), \quad \forall 0 \leq t \leq T_{0},
$$

$$
\frac{\mathrm{d}}{\mathrm{d} t} y(t)+\frac{\varepsilon}{K_{p}} z(t) \leq 0, \quad \forall 0 \leq t \leq T_{0} .
$$

Noting that $\lambda_{0}^{2} y(t) \leq z(t)$, where $\lambda_{0}$ is the first positive eigenvalue of the operator $(-\Delta)$ with Neumann boundary conditions, we get

$$
\frac{\mathrm{d}}{\mathrm{d} t} y(t)+\gamma y(t) \leq 0, \quad \forall 0 \leq t \leq T_{0},
$$

where $\gamma=\frac{\varepsilon \lambda_{0}^{2}}{K_{p}}$. We obtain that $y(t) \leq y(0) \mathrm{e}^{-\gamma t}, \forall 0 \leq t<T_{0}$. In particular $y(t) \leq \delta$ so that for all $t \leq T_{0}$, so using (53) we have

$$
K_{\alpha}\left(z^{\frac{3}{8}} y^{\frac{1}{8}}+z^{\frac{5}{6}} y^{\frac{1}{6}}+z^{\frac{8-17 \alpha}{16}} y^{\frac{\alpha}{16}}+z^{\frac{2-\alpha}{4}} y^{\frac{\alpha}{4}}\right) \leq \frac{\varepsilon}{4 K_{p}}, \quad \forall 0 \leq t<T_{0} .
$$

Finally, thanks to the continuity of $y$ in time and the maximality of $T_{0}$ satisfying the property (54), we can conclude that in fact $T_{0}=+\infty$ and the claim is proved.

In the same way, the proof of Theorem 3.9 for $d=3$ is the following.

Proof. We consider here the case where $\Lambda=0$, that is $F=F_{0}$. We use the same approach than before and we get in this particular case

$$
\begin{aligned}
\frac{1}{2} \frac{\mathrm{d}}{\mathrm{d} t}\left((-\Delta)^{-1} c_{1}, c_{1}\right)_{\mathrm{L}^{2}}+\frac{3 M_{0} \varepsilon}{4}\left|\nabla c_{1}\right|_{\mathrm{L}^{2}}^{2}+\frac{12 M_{0}}{\varepsilon} \int_{\Omega} c_{1}^{2}\left(1-\beta_{1}\right. & \left.\left(c_{2}+\frac{c_{1}}{2}\right)\left(1-\left(c_{2}+\frac{c_{1}}{2}\right)\right)\right) \mathrm{d} x \\
& =\frac{12 M_{0}}{\varepsilon} \int_{\Omega} c_{1}^{3}\left(3-\frac{\beta_{1}}{2}\right)+c_{1}^{4}\left(\frac{\beta_{1}}{4}-2\right) \mathrm{d} x
\end{aligned}
$$

where we have written $c_{2}=c_{2}+\frac{c_{1}}{2}-\frac{c_{1}}{2}$, and $c_{3}=1-c_{1}-c_{2}=1-\left(c_{2}+\frac{c_{1}}{2}\right)-\frac{c_{1}}{2}$.

As $\Sigma_{i}>\frac{\Sigma_{T}}{2}>0$, we see from (49) that $\beta_{1}<4$. Therefore, we have $1-\beta_{1} X(1-X)>1-\frac{\beta_{1}}{4}>0$, for all $X \in \mathbb{R}$. Then, the integral term in the left-hand side of (55) is non-negative. Hence, using Young's inequality we obtain

$$
\frac{1}{2} \frac{\mathrm{d}}{\mathrm{d} t}\left((-\Delta)^{-1} c_{1}, c_{1}\right)_{\mathrm{L}^{2}}+\frac{3 M_{0} \varepsilon}{4}\left|\nabla c_{1}\right|_{\mathrm{L}^{2}}^{2} \leq K\left(\left|c_{1}\right|_{\mathrm{L}^{3}}^{3}+\left|c_{1}\right|_{\mathrm{L}^{4}}^{4}\right)
$$


Then we use the embedding $\mathrm{H}^{\frac{3}{4}} \subset \mathrm{L}^{4}$, the interpolation $\mathrm{H}^{\frac{3}{4}}=\left[\mathrm{H}^{1}, \mathrm{~L}^{2}\right]_{\frac{1}{4}}$ and the inequality (47) in order to conclude by the same argument as in the previous proof.

\section{Proof of Theorem 2.4}

We turn back in this section to the proof of our well-posedness result for the system (8). We recall that we have to take care of the fact that one of the coefficients $\left(\Sigma_{i}\right)_{i}$ is allowed to be negative (as soon as the assumptions of the theorem are fulfilled).

As usual, the various constants depending only on the physical parameters of the problem, appearing in the following proof may change from one line to another without changing the notation. Furthermore, we only give the proof in the $3 \mathrm{D}$ case, which is more difficult than the $2 \mathrm{D}$ one.

Proof.

- Step 1. Galerkin approximation

We introduce the family $\left(\varphi^{\ell}\right)_{\ell \geq 1}$ of the eigenfunctions of the operator $-\Delta$ (with homogeneous Neumann condition). We assume that $\varphi^{1}=1$. We recall that the functions $\left(\varphi^{\ell}\right)_{\ell}$ are orthogonal in $\mathrm{L}^{2}$ and $\mathrm{H}^{1}$. Let $\Phi^{n}=\operatorname{Span}\left(\varphi^{1}, \ldots, \varphi^{n}\right)$ the space spanned by the family $\left(\varphi^{\ell}\right)_{1<\ell<n}$ and $P_{\Phi^{n}}$ the orthogonal projector on $\Phi^{n}$ in $\mathrm{L}^{2}(\Omega)$.

We look for $\left(\mathbf{C}^{n}, \boldsymbol{\mu}^{n}\right)$ defined by for $i=\{1,2,3\}$

$$
c_{i}^{n}=\sum_{\ell=1}^{n} \alpha_{i}^{\ell}(t) \varphi^{\ell} \quad \mu_{i}^{n}=\sum_{\ell=1}^{n} \beta_{i}^{\ell}(t) \varphi^{\ell}
$$

where $\alpha_{i}^{\ell}$ and $\beta_{i}^{\ell}$ are smooth, such that $\mathbf{C}^{n}(0)=P_{\Phi^{n}}\left(\mathbf{C}^{0}\right)$ and

$$
\begin{gathered}
\left(\frac{\mathrm{d}}{\mathrm{d} t} c_{i}^{n}, \varphi\right)=-\int_{\Omega} \frac{M_{0}}{\Sigma_{i}} \nabla \mu_{i}^{n} \cdot \nabla \varphi \mathrm{d} x, \quad \forall \varphi \in \Phi^{n}, \quad \forall i \in\{1,2,3\}, \\
\int_{\Omega} \mu_{i}^{n} \varphi \mathrm{d} x=\int_{\Omega} \frac{3}{4} \varepsilon \Sigma_{i} \nabla c_{i}^{n} \cdot \nabla \varphi+\frac{4 \Sigma_{T}}{\varepsilon} \sum_{j \neq i}\left[\frac{1}{\Sigma_{j}}\left(\partial_{i} F\left(\mathbf{C}^{n}\right)-\partial_{j} F\left(\mathbf{C}^{n}\right)\right)\right] \varphi \mathrm{d} x, \quad \forall \varphi \in \Phi^{n}, \quad \forall i \in\{1,2,3\} .
\end{gathered}
$$

The approximate problem (56) is a system of ordinary differential equations where the unknowns are the functions $\alpha_{i}^{\ell}$ since the equation (57) gives $\beta_{i}^{\ell}$ as a nonlinear function of the $\left(\alpha_{i}^{\ell}\right)_{\ell}$. Since the bulk free energy $F$ is smooth enough we can use the Cauchy-Lipschitz theorem which says that there exists a unique solution of problem (56)-(57) on a maximal time interval $\left[0, t^{n}\left[, t^{n} \in\right] 0,+\infty\right]$.

Let us remark that, as $\mathbf{C}^{0} \in \mathcal{S}$, we have $c_{1}(0)+c_{2}(0)+c_{3}(0)=1$, and then

$$
P_{\Phi^{n}} c_{1}^{n}(0)+P_{\Phi^{n}} c_{2}^{n}(0)+P_{\Phi^{n}} c_{3}^{n}(0)=P_{\Phi^{n}} 1=1
$$

because $\varphi^{1}=1$ belongs to all the Galerkin approximation spaces. Furthermore, we have seen that the system (8) is built to ensure that for any $t \in\left[0, t^{n}\right]$

$$
\sum_{i=1}^{3} c_{i}^{n}=1
$$

Hence, $\mathbf{C}^{n}(t) \in \mathcal{S}$ for any $n$, and any $t \in\left[0, t^{n}[\right.$.

- Step 2. Mass conservation

From (56) with $\varphi=\varphi_{1}$ we find that

$$
\frac{\mathrm{d}}{\mathrm{d} t} m\left(c_{i}^{n}\right)=\frac{1}{|\Omega|} \frac{\mathrm{d}}{\mathrm{d} t} \int_{\Omega} c_{i}^{n}(t) \varphi_{1} \mathrm{~d} x=0 .
$$


Hence for any $t \in\left[0, t^{n}[\right.$, we have

$$
m\left(c_{i}^{n}(t)\right)=m\left(c_{i}^{n}(0)\right)=m\left(P_{\Phi^{n}}\left(c_{i}^{0}\right)\right)=m\left(c_{i}^{0}\right) .
$$

- Step 3. Energy estimates

Take $\varphi=\mu_{i}^{n}$ in (56) and $\varphi=\frac{\mathrm{d} c_{i}^{n}}{\mathrm{~d} t}$ in (57). We obtain for any $i \in\{1,2,3\}$,

$$
\left(\frac{\mathrm{d} c_{i}^{n}}{\mathrm{~d} t}, \mu_{i}^{n}\right)=-\int_{\Omega} \frac{M_{0}}{\Sigma_{i}}\left|\nabla \mu_{i}^{n}\right|^{2} \mathrm{~d} x
$$

and

$$
\left(\frac{\mathrm{d} c_{i}^{n}}{\mathrm{~d} t}, \mu_{i}^{n}\right)=\int_{\Omega} \frac{3}{4} \varepsilon \Sigma_{i} \nabla c_{i}^{n} \cdot \nabla \frac{\mathrm{d} c_{i}^{n}}{\mathrm{~d} t}+\frac{4 \Sigma_{T}}{\varepsilon} \sum_{j \neq i}\left[\frac{1}{\Sigma_{j}}\left(\partial_{i} F\left(\mathbf{C}^{n}\right)-\partial_{j} F\left(\mathbf{C}^{n}\right)\right)\right] \frac{\mathrm{d} c_{i}^{n}}{\mathrm{~d} t} \mathrm{~d} x .
$$

Summing these equations over $i$ from 1 to 3 and using the property $\frac{\mathrm{d}}{\mathrm{d} t} \sum_{i=1}^{3} c_{i}^{n}=0$ we get

$$
\frac{\mathrm{d}}{\mathrm{d} t}\left[\int_{\Omega} \sum_{i=1}^{3} \frac{3}{8} \varepsilon \Sigma_{i}\left|\nabla c_{i}^{n}\right|^{2} \mathrm{~d} x+\frac{12}{\varepsilon} \int_{\Omega} F\left(\mathbf{C}^{n}\right) \mathrm{d} x\right]+\sum_{i=1}^{3} \int_{\Omega} \frac{M_{0}}{\Sigma_{i}}\left|\nabla \mu_{i}^{n}\right|^{2} \mathrm{~d} x=0 .
$$

Of course, the term under the time derivative is nothing but the total energy of the system $\mathcal{F}_{\boldsymbol{\Sigma}, \varepsilon}^{\text {triph }}\left(\mathbf{C}^{n}(t)\right)$. Using Proposition 2.1 and the property (9), we see that the second term in (60) satisfies

$$
\sum_{i=1}^{3} \int_{\Omega} \frac{M_{0}}{\Sigma_{i}}\left|\nabla \mu_{i}^{n}\right|^{2} \mathrm{~d} x=\sum_{i=1}^{3} \int_{\Omega} M_{0} \Sigma_{i}\left|\frac{\nabla \mu_{i}^{n}}{\Sigma_{i}}\right|^{2} \mathrm{~d} x \geq \underline{\Sigma} M_{0} \sum_{i=1}^{3} \frac{1}{\Sigma_{i}^{2}}\left|\nabla \mu_{i}\right|_{\mathrm{L}^{2}}^{2} .
$$

In particular this term is non-negative and we finally find that the total energy of the system decreases along the time. Furthermore, we can control the energy of the approximate initial data as follows

$$
\begin{aligned}
\int_{\Omega} \sum_{i=1}^{3} \frac{3}{8} \varepsilon \Sigma_{i}\left|\nabla c_{i}^{n}(0)\right|^{2} \mathrm{~d} x+\frac{12}{\varepsilon} \int_{\Omega} F\left(\mathbf{C}^{n}(0)\right) \mathrm{d} x & \leq \sum_{i=1}^{3} \frac{3}{8} \varepsilon\left|\Sigma_{i}\right|\left|\nabla c_{i}^{0}\right|_{\mathrm{L}^{2}}^{2}+\frac{12}{\varepsilon}\left(B_{1}\left|\mathbf{C}^{n}(0)\right|_{\mathrm{L}^{6}}^{6}+B_{2}|\Omega|\right) \\
& \leq K_{1}+K_{2} \sum_{i=1}^{3}\left|P_{\Phi^{n}} c_{i}^{0}\right|_{\mathrm{H}^{1}}^{6} \leq K_{1}+K_{2}\left|\mathbf{C}^{0}\right|_{\mathrm{H}^{1}}^{6},
\end{aligned}
$$

since we assumed that $p=6$ in (14) as soon as $d=3$.

We have shown a bound on the total energy $\mathcal{F}_{\boldsymbol{\Sigma}, \varepsilon}^{\text {triph }}\left(\mathbf{C}^{n}(t)\right)$ which does not depend on $t$ and $n$. Using Proposition 2.1 (and (58)) and assumption (13), we find that the $\mathrm{H}^{1}(\Omega)$ semi-norm of $\mathbf{C}^{n}(t)$ is controlled by the total energy. As a consequence, with (59), we get that the existence time of the approximate problem is $t^{n}=+\infty$ and that the following estimate holds

$$
\left|\mathbf{C}^{n}\right|_{L^{\infty}\left(0, \infty ; \mathrm{H}^{1}\right)} \leq K_{1} .
$$

Coming back to (60) and using (61) we find that

$$
\left|\nabla \boldsymbol{\mu}^{n}\right|_{\mathrm{L}^{2}\left(0, \infty ; \mathrm{L}^{2}\right)} \leq K_{1} .
$$

Furthermore, there exists $K_{2}>0$ independent of $\mathrm{t}$ and of $\mathrm{n}$ such that

$$
\left|m\left(\mu_{i}^{n}\right)\right|<K_{2}, \quad \forall i \in\{1,2,3\} .
$$


Indeed, we have

$$
\begin{aligned}
m\left(\mu_{i}^{n}\right)=\left(\mu_{i}^{n}, \varphi_{1}\right) & =\frac{3}{4} \varepsilon \Sigma_{i}\left(-\Delta c_{i}^{n}, \varphi_{1}\right)+\left(\frac{4 \Sigma_{T}}{\varepsilon} \sum_{j \neq i}\left(\frac{1}{\Sigma_{j}}\left(\partial_{i} F\left(\mathbf{C}^{n}\right)-\partial_{j} F\left(\mathbf{C}^{n}\right)\right)\right), \varphi_{1}\right) \\
& =\int_{\Omega} \frac{4 \Sigma_{T}}{\varepsilon} \sum_{j \neq i}\left(\frac{1}{\Sigma_{j}}\left(\partial_{i} F\left(\mathbf{C}^{n}\right)-\partial_{j} F\left(\mathbf{C}^{n}\right)\right)\right) \mathrm{d} x,
\end{aligned}
$$

thanks to the Neumann boundary condition $\nabla c_{i}^{n} \cdot n=0$ on $\Gamma$. Using (15), we have

$$
\begin{aligned}
\left|m\left(\mu_{i}^{n}\right)\right| & \leq \frac{4\left|\Sigma_{T}\right|}{\varepsilon} \int_{\Omega} \sum_{j \neq i}\left(\frac{1}{\left|\Sigma_{j}\right|}\left(\left|\partial_{i} F\left(\mathbf{C}^{n}\right)\right|+\left|\partial_{j} F\left(\mathbf{C}^{n}\right)\right|\right)\right) \mathrm{d} x \\
& \leq K\left(\int_{\Omega} B_{1}\left|\mathbf{C}^{n}\right|^{p-1} \mathrm{~d} x+B_{2}|\Omega|\right) \leq K\left(1+\left|\mathbf{C}^{n}\right|_{\mathrm{H}^{1}}^{5}\right),
\end{aligned}
$$

because $p=6$ in (15) as soon as $d=3$. The estimate (64) follows from (62). Finally, combining (63) and (64) we get, by the Poincaré inequality, for any $n$

$$
\left|\boldsymbol{\mu}^{n}\right|_{\mathrm{L}^{2}\left(0, T ; \mathrm{H}^{1}\right)}<K_{3}(1+T), \quad \forall T>0 .
$$

In order to establish a compactness property for the approximate solutions under study, we now look for an estimate of $\frac{\partial \mathbf{C}^{n}}{\partial t}$ in the space $\mathrm{L}^{2}\left(0, T ;\left(\mathrm{H}^{-1}(\Omega)\right)^{3}\right)$. From equation (56) we deduce

$$
\begin{aligned}
\left|\int_{0}^{T}\left\langle\frac{\partial c_{i}^{n}}{\partial t}, \varphi\right\rangle_{\mathrm{H}^{-1}, \mathrm{H}_{0}^{1}} \mathrm{~d} t\right| & =\left|\int_{0}^{T} \int_{\Omega} \frac{M_{0}}{\Sigma_{i}} \nabla \mu_{i}^{n} \cdot \nabla P_{\Phi^{n}} \varphi \mathrm{d} x \mathrm{~d} t\right|=\left|\int_{0}^{T} \int_{\Omega} \frac{M_{0}}{\Sigma_{i}} \nabla \mu_{i}^{n} \cdot \nabla \varphi \mathrm{d} x \mathrm{~d} t\right| \\
& \leq K\left|\nabla \mu_{i}^{n}\right|_{\mathrm{L}^{2}\left(0, T ; \mathrm{L}^{2}\right)}|\nabla \varphi|_{\mathrm{L}^{2}\left(0, T ; \mathrm{L}^{2}\right)} \leq K|\nabla \varphi|_{\mathrm{L}^{2}\left(0, T ; \mathrm{L}^{2}\right)}, \forall i \in\{1,2,3\}
\end{aligned}
$$

for any $\varphi \in \mathrm{L}^{2}\left(0, T ; \mathrm{H}_{0}^{1}(\Omega)\right)$, thanks to estimate (63). It follows that

$$
\left|\frac{\partial \mathbf{C}^{n}}{\partial t}\right|_{\mathrm{L}^{2}\left(0, T ; \mathrm{H}^{-1}\right)}<K .
$$

- Step 4. Passage to the limit in the equations

Using the estimates (62), (65) and (66), we can extract subsequences of $\left(\mathbf{C}^{n}\right)_{n}$ and $\left(\boldsymbol{\mu}^{n}\right)_{n}$ (always denoted by $\left(\mathbf{C}^{n}\right)_{n}$ and $\left.\left(\boldsymbol{\mu}^{n}\right)_{n}\right)$ such that

$$
\begin{array}{llll}
\mathbf{C}^{n} & \rightarrow & \mathbf{C} & \text { in } \mathrm{L}^{\infty}\left(0, \infty,\left(\mathrm{H}^{1}(\Omega)\right)^{3}\right) \text { weak-* } \\
\mathbf{C}^{n} \rightarrow & \mathbf{C} & \text { in } \mathrm{L}_{l o c}^{2}\left(0, \infty,\left(\mathrm{H}^{1}(\Omega)\right)^{3}\right) \text { weak } \\
\frac{\partial \mathbf{C}^{n}}{\partial t} \rightarrow \frac{\partial \mathbf{C}}{\partial t} & \text { in } \mathrm{L}_{l o c}^{2}\left(0, \infty,\left(\mathrm{H}^{-1}(\Omega)\right)^{3}\right) \text { weak } \\
\boldsymbol{\mu}^{n} \rightarrow \boldsymbol{\mu} & \text { in } \mathrm{L}_{l o c}^{2}\left(0, \infty,\left(\mathrm{H}^{1}(\Omega)\right)^{3}\right) \text { weak. }
\end{array}
$$

From estimates (62) and (66), we can use the Aubin-Lions-Simon's compactness theorem to obtain, up to a subsequence,

$$
\mathbf{C}^{n} \rightarrow \mathbf{C} \text { in } \mathcal{C}^{0}\left(\left[0, T\left[,\left(\mathrm{~L}^{p}(\Omega)\right)^{3}\right) \text { strong, } \forall T>0, \forall p<6\right.\right.
$$

In particular, $\mathbf{C}^{n}(0)$ converges strongly to $\mathbf{C}(0)$ in $\left(\mathrm{L}^{2}(\Omega)\right)^{3}$ and thus $\mathbf{C}(0)=\mathbf{C}^{0}$ because $P_{\Phi^{n}}$ converges to the identity for the strong topology of operators.

It is now straightforward, using the above convergences, to pass to the limit in the approximate problem. This shows the existence of a weak solution to problem (8). 
- Step 5. Further estimates

Let $(\mathbf{C}, \boldsymbol{\mu})$ be the weak solution to (8) obtained in the previous step. We already know that $\mathbf{C}$ belongs to $\mathrm{L}^{\infty}\left(0,+\infty,\left(\mathrm{H}^{1}(\Omega)\right)^{3}\right)$ and that $\nabla \boldsymbol{\mu}$ belongs to $\mathrm{L}^{2}\left(0,+\infty,\left(\mathrm{L}^{2}(\Omega)\right)^{9}\right)$. We want now to investigate further regularity properties of $\mathbf{C}$. The following estimates will be fundamental in order to prove the uniqueness of such a solution in the next step.

First, we seek estimates for $\Delta \mathbf{C}$ in $\left(L^{2}(\Omega)\right)^{3}$. Multiplying by $-\Delta c_{i}$ the definition of $\mu_{i}$ in (8), and integrating by parts, we get for each $i \in\{1,2,3\}$

$$
\left(\nabla \mu_{i}, \nabla c_{i}\right)=\frac{3}{4} \varepsilon \Sigma_{i}\left|\Delta c_{i}\right|_{\mathrm{L}^{2}}^{2}-\frac{4 \Sigma_{T}}{\varepsilon} \int_{\Omega} \sum_{j \neq i}\left[\frac{1}{\Sigma_{j}}\left(\partial_{i} F(\mathbf{C})-\partial_{j} F(\mathbf{C})\right)\right] \Delta c_{i} \mathrm{~d} x
$$

Summing these equations over $i$ and noting that, thanks to (58), $\sum_{i=1}^{3} \Delta c_{i}=0$, one gets

$$
\sum_{i=1}^{3}\left(\nabla \mu_{i}, \nabla c_{i}\right)=\sum_{i=1}^{3} \frac{3}{4} \varepsilon \Sigma_{i}\left|\Delta c_{i}\right|_{\mathrm{L}^{2}}^{2}+\frac{12}{\varepsilon} \sum_{i=1}^{3} \int_{\Omega} \nabla \partial_{i} F(\mathbf{C}) \cdot \nabla c_{i} \mathrm{~d} x .
$$

Let us pay attention to the last term $V$ above. Thanks to assumption (17) and to the mass conservation property, this term satisfies

$$
\begin{aligned}
V & =\frac{12}{\varepsilon} \sum_{i=1}^{3} \sum_{j=1}^{3} \sum_{k=1}^{d} \int_{\Omega} \partial_{i} \partial_{j} F(\mathbf{C}) \partial_{x_{k}} c_{j} \partial_{x_{k}} c_{i} \mathrm{~d} x \\
& =\frac{12}{\varepsilon} \sum_{k=1}^{d} \int_{\Omega}\left(D^{2} F(\mathbf{C}) \cdot \partial_{x_{k}} \mathbf{C}, \partial_{x_{k}} \mathbf{C}\right) \mathrm{d} x \\
& \geq-\frac{12}{\varepsilon} D_{1} \sum_{k=1}^{d} \int_{\Omega}\left(1+|\mathbf{C}|^{q}\right)\left|\partial_{x_{k}} \mathbf{C}\right|^{2} \mathrm{~d} x \geq-\frac{K}{\varepsilon} \int_{\Omega}\left(1+|\mathbf{C}-m(\mathbf{C})|^{q}\right)|\nabla \mathbf{C}|^{2} \mathrm{~d} x .
\end{aligned}
$$

Using Proposition 2.1, we deduce from (67) that

$$
\varepsilon \underline{\Sigma}|\Delta \mathbf{C}|_{\mathrm{L}^{2}}^{2} \leq \sum_{i=1}^{3} \varepsilon \Sigma_{i}\left|\Delta c_{i}^{n}\right|_{\mathrm{L}^{2}}^{2} \leq \frac{K}{\varepsilon} \int_{\Omega}\left(1+|\mathbf{C}-m(\mathbf{C})|^{q}\right)|\nabla \mathbf{C}|^{2} \mathrm{~d} x+K|\nabla \boldsymbol{\mu}|_{\mathrm{L}^{2}}|\nabla \mathbf{C}|_{\mathrm{L}^{2}} .
$$

As $d=3$, using the elliptic regularity of the Laplace operator and Agmon and Poincaré inequalities, we get

$$
\int_{\Omega}|\mathbf{C}-m(\mathbf{C})|^{q}|\nabla \mathbf{C}|^{2} \mathrm{~d} x \leq|\mathbf{C}-m(\mathbf{C})|_{L^{\infty}}^{q}|\nabla \mathbf{C}|_{\mathrm{L}^{2}}^{2} \leq K|\Delta \mathbf{C}|_{L^{2}}^{\frac{q}{2}}|\nabla \mathbf{C}|_{\mathrm{L}^{2}}^{\frac{q}{2}+2} .
$$

Since in the assumption (17) for the three-dimensional case we assumed that $q<4$, we see that the power of $|\Delta \mathbf{C}|_{L^{2}}$ in the above inequality is strictly less than 2. Hence, using Young's inequality it follows from (68)

$$
|\Delta \mathbf{C}|_{\mathrm{L}^{2}}^{2} \leq K\left(1+|\nabla \mathbf{C}|_{\mathrm{L}^{2}}^{2\left(\frac{4+q}{4-q}\right)}\right)+|\nabla \boldsymbol{\mu}|_{\mathrm{L}^{2}}|\nabla \mathbf{C}|_{\mathrm{L}^{2}} .
$$

Since $\mathbf{C} \in L^{\infty}\left(0,+\infty,\left(\mathrm{H}^{1}(\Omega)\right)^{3}\right)$ and $\nabla \boldsymbol{\mu} \in L^{2}\left(0,+\infty,\left(\mathrm{L}^{2}(\Omega)\right)^{9}\right)$, we see that the right-hand side of this estimate belongs to $L_{l o c}^{2}(] 0,+\infty[)$. It follows that

$$
|\mathbf{C}|_{\mathrm{L}^{4}\left(0, T ; \mathrm{H}^{2}\right)} \leq K(T), \quad \forall 0 \leq T<+\infty .
$$


We can now show, as claimed in Theorem 2.4, that $\mathbf{C}$ belongs to $\mathrm{L}^{2}\left(0, T ;\left(\mathrm{H}^{3}(\Omega)\right)^{3}\right)$, for any $T>0$. Indeed, taking the gradient of the definition of the chemical potential $\mu_{i}$ we get

$$
\frac{3}{4} \varepsilon \Sigma_{i} \nabla \Delta c_{i}=\nabla \mu_{i}-\nabla\left(\frac{4 \Sigma_{T}}{\varepsilon} \sum_{j \neq i}\left[\frac{1}{\Sigma_{j}}\left(\partial_{i} F(\mathbf{C})-\partial_{j} F(\mathbf{C})\right)\right]\right) .
$$

Hence,

$$
\varepsilon^{2} \sum_{i=1}^{3} \Sigma_{i}^{2}\left|\nabla \Delta c_{i}\right|_{\mathrm{L}^{2}}^{2} \leq K|\nabla \boldsymbol{\mu}|_{\mathrm{L}^{2}}^{2}+K \sum_{i=1}^{3}\left|\nabla \partial_{i} F(\mathbf{C})\right|_{\mathrm{L}^{2}}^{2} .
$$

Since $d=3$, we use (16) and the following Agmon's inequality

$$
|\nabla \mathbf{C}|_{\infty} \leq K|\Delta \mathbf{C}|_{L^{2}}^{\frac{1}{2}}|\nabla \Delta \mathbf{C}|_{L^{2}}^{\frac{1}{2}}
$$

in order to obtain

$$
\begin{aligned}
\sum_{i=1}^{3}\left|\nabla \partial_{i} F(\mathbf{C})\right|_{\mathrm{L}^{2}}^{2} & \leq \sum_{i, j=1}^{3}\left|\partial_{i} \partial_{j} F(\mathbf{C}) \nabla \mathbf{C}\right|_{\mathrm{L}^{2}}^{2} \leq K \int_{\Omega}\left(1+|\mathbf{C}|^{p-2}\right)^{2}|\nabla \mathbf{C}|^{2} \mathrm{~d} x \\
& \leq K|\nabla \mathbf{C}|_{\infty}^{2}\left(1+|\mathbf{C}|_{\mathrm{L}^{2(p-2)}}^{2(p-2)}\right) \leq K|\Delta \mathbf{C}|_{\mathrm{L}^{2}}|\nabla \Delta \mathbf{C}|_{\mathrm{L}^{2}}\left(1+|\mathbf{C}|_{\mathrm{L}^{2(p-2)}}^{2(p-2)}\right) \\
& =K|\Delta \mathbf{C}|_{\mathrm{L}^{2}}|\nabla \Delta \mathbf{C}|_{\mathrm{L}^{2}}\left(1+|\mathbf{C}|_{\mathrm{L}^{8}}^{8}\right)
\end{aligned}
$$

because, in the $3 \mathrm{D}$ case, we assumed that $p=6$ in (16). Using now the embedding $\mathrm{H}^{\frac{9}{8}} \subset \mathrm{L}^{8}$ and the interpolation property $\mathrm{H}^{\frac{9}{8}}=\left[\mathrm{H}^{2}, \mathrm{H}^{1}\right]_{\frac{7}{8}}$, we deduce that

$$
\begin{aligned}
K \sum_{i=1}^{3}\left|\nabla \partial_{i} F(\mathbf{C})\right|_{\mathrm{L}^{2}}^{2} & \leq K|\Delta \mathbf{C}|_{\mathrm{L}^{2}}|\nabla \Delta \mathbf{C}|_{\mathrm{L}^{2}}\left(1+|\mathbf{C}|_{\mathrm{H}^{1}}^{7}|\mathbf{C}|_{\mathrm{H}^{2}}\right) \\
& \leq \frac{\varepsilon^{2}}{2} \sum_{i=1}^{3} \Sigma_{i}^{2}\left|\nabla \Delta c_{i}\right|_{\mathrm{L}^{2}}^{2}+K|\Delta \mathbf{C}|_{\mathrm{L}^{2}}^{2}\left(1+|\mathbf{C}|_{\mathrm{H}^{1}}^{14}|\mathbf{C}|_{\mathrm{H}^{2}}^{2}\right) .
\end{aligned}
$$

Using this last estimate in (71), we get

$$
\frac{\varepsilon^{2}}{2} \sum_{i=1}^{3} \Sigma_{i}^{2}\left|\nabla \Delta c_{i}\right|_{\mathrm{L}^{2}}^{2} \leq K|\nabla \boldsymbol{\mu}|_{\mathrm{L}^{2}}^{2}+K|\mathbf{C}|_{\mathrm{H}^{2}}^{2}\left(1+|\mathbf{C}|_{\mathrm{H}^{1}}^{14}|\mathbf{C}|_{\mathrm{H}^{2}}^{2}\right) .
$$

We remark that the power of $|\mathbf{C}|_{\mathrm{H}^{2}}$ in this estimate is exactly equal to 4 so that, with (62), (65), (70), we obtain

$$
|\mathbf{C}|_{\mathrm{L}^{2}\left(0, T ; \mathrm{H}^{3}\right)} \leq K(T), \quad \forall 0 \leq T<+\infty,
$$

using once more the elliptic regularity properties of the Laplace operator.

- Step 6. Uniqueness

We assume that there exist two weak solutions $\left(c_{i}, \mu_{i}\right)$ and $\left(d_{i}, \nu_{i}\right)$ for $i=1,2,3$ of problem (8) for the same initial data. Letting $\mathbf{C}=\left(c_{1}, c_{2}, c_{3}\right)$ and $\mathbf{D}=\left(d_{1}, d_{2}, d_{3}\right)$, we have for all $\varphi \in \mathrm{H}^{1}$,

$$
\left(\frac{\partial}{\partial t}\left(c_{i}-d_{i}\right), \varphi\right)=-\frac{M_{0}}{\Sigma_{i}} \int_{\Omega}\left(\nabla \mu_{i}-\nabla \nu_{i}\right) \cdot \nabla \varphi \mathrm{d} x
$$


Taking $\varphi=\varepsilon \Sigma_{i}\left(c_{i}-d_{i}\right)$ as a test function and summing the equations over $i$ leads to

$$
\begin{aligned}
\frac{\mathrm{d}}{\mathrm{d} t}\left(\sum_{i=1}^{3} \frac{\varepsilon \Sigma_{i}}{2}\left|c_{i}-d_{i}\right|_{\mathrm{L}^{2}}^{2}\right) & =-\varepsilon M_{0} \sum_{i=1}^{3} \int_{\Omega} \nabla\left(\mu_{i}-\nu_{i}\right) \cdot \nabla\left(c_{i}-d_{i}\right) \mathrm{d} x \\
& =\varepsilon M_{0} \sum_{i=1}^{3} \int_{\Omega}\left(\mu_{i}-\nu_{i}\right) \Delta\left(c_{i}-d_{i}\right) \mathrm{d} x \underset{\text { def }}{=} U .
\end{aligned}
$$

We are now going to estimate the right-hand side $U$ of this equality. Using the definition of $\mu_{i}$ and $\nu_{i}$ and noting that $\sum_{i=1}^{3} \Delta\left(c_{i}-d_{i}\right)=0$, one gets

$$
U=-\varepsilon^{2} \sum_{i=1}^{3} \frac{3}{4} M_{0} \Sigma_{i}\left|\Delta\left(c_{i}-d_{i}\right)\right|_{\mathrm{L}^{2}}^{2}+12 M_{0} \sum_{i=1}^{3} \int_{\Omega}\left(\partial_{i} F(\mathbf{C})-\partial_{i} F(\mathbf{D})\right) \Delta\left(c_{i}-d_{i}\right) \mathrm{d} x .
$$

Using Proposition 2.1, Young's inequality and assumption (16), we deduce that

$$
\begin{aligned}
U+\frac{\Sigma}{2} \varepsilon^{2} M_{0} \sum_{i=1}^{3}\left|\Delta\left(c_{i}-d_{i}\right)\right|_{\mathrm{L}^{2}}^{2} & \leq K \sum_{i=1}^{3} \int_{\Omega}\left|\partial_{i} F(\mathbf{C})-\partial_{i} F(\mathbf{D})\right|^{2} \mathrm{~d} x \\
& \leq K \int_{\Omega}\left(1+|\mathbf{C}|^{2(p-2)}+|\mathbf{D}|^{2(p-2)}\right)|\mathbf{C}-\mathbf{D}|^{2} \mathrm{~d} x \\
& \leq K\left(1+|\mathbf{C}|_{\mathrm{L}^{2(p-2)}}^{2(p-2)}+|\mathbf{D}|_{\mathrm{L}^{2(p-2)}}^{2(p-2)}\right)|\mathbf{C}-\mathbf{D}|_{\mathrm{L}^{\infty}}^{2}
\end{aligned}
$$

Since we only consider in this proof the three dimensional case, Agmon and Young inequalities yield

$$
\begin{aligned}
U+K_{1} \Sigma \varepsilon^{2}|\Delta(\mathbf{C}-\mathbf{D})|_{\mathrm{L}^{2}}^{2} & \leq K\left(1+|\mathbf{C}|_{\mathrm{L}^{2(p-2)}}^{8(p-2)}+|\mathbf{D}|_{\mathrm{L}^{2(p-2)}}^{8(p-2)}\right)|\mathbf{C}-\mathbf{D}|_{\mathrm{L}^{2}}^{2} \\
& =K\left(1+|\mathbf{C}|_{\mathrm{L}^{8}}^{32}+|\mathbf{D}|_{\mathrm{L}^{8}}^{32}\right)|\mathbf{C}-\mathbf{D}|_{\mathrm{L}^{2}}^{2},
\end{aligned}
$$

because we assumed that $p=6$ in (16) when $d=3$. We now use the embedding $\mathrm{H}^{\frac{9}{8}} \subset \mathrm{L}^{8}$ and the interpolation property $\mathrm{H}^{\frac{9}{8}}=\left[\mathrm{H}^{3}, \mathrm{H}^{1}\right]_{\frac{15}{16}}$ to get

It follows that

$$
|\mathbf{C}|_{\mathrm{L}^{8}}^{32} \leq K|\mathbf{C}|_{\mathrm{H}^{1}}^{30}|\mathbf{C}|_{\mathrm{H}^{3}}^{2}
$$

$$
U \leq K\left[1+|\mathbf{C}|_{\mathrm{H}^{1}}^{30}|\mathbf{C}|_{\mathrm{H}^{3}}^{2}+|\mathbf{D}|_{\mathrm{H}^{1}}^{30}|\mathbf{D}|_{\mathrm{H}^{3}}^{2}\right]|\mathbf{C}-\mathbf{D}|_{\mathrm{L}^{2}}^{2} .
$$

We can now proceed to the proof of the uniqueness. Let us introduce

$$
y(t)=\sum_{i=1}^{3} \frac{\varepsilon \Sigma_{i}}{2}\left|c_{i}-d_{i}\right|_{\mathrm{L}^{2}}^{2} .
$$

Equation (74) and estimate (75) leads to the differential inequality

$$
y^{\prime}(t) \leq a(t)|\mathbf{C}-\mathbf{D}|_{\mathrm{L}^{2}}^{2},
$$

where $a$ is the function defined by

$$
a(t)=K\left[1+|\mathbf{C}|_{\mathrm{H}^{1}}^{30}|\mathbf{C}|_{\mathrm{H}^{3}}^{2}+|\mathbf{D}|_{\mathrm{H}^{1}}^{30}|\mathbf{D}|_{\mathrm{H}^{3}}^{2}\right] .
$$


Using now Proposition 2.1 (and since $\sum_{i=1}^{3}\left(c_{i}-d_{i}\right)=0$ ) we deduce that $y$ is a non-negative function and that

$$
y^{\prime}(t) \leq \frac{2}{\underline{\Sigma} \varepsilon} a(t) y(t)
$$

Thanks to the regularity properties (18) of the solutions $\mathbf{C}$ and $\mathbf{D}$, we see that $a$ lies in $L^{1}(] 0, T[)$ for any $T>0$. Furthermore, $y(0)=0$ since $\mathbf{C}$ and $\mathbf{D}$ have the same initial data. Using the Gronwall lemma we deduce that $y(t)=0$ for any positive time which proves that $\mathbf{C}=\mathbf{D}$.

\section{Numerical Simulations}

First of all, we expose in this section the fully coupled Navier-Stokes/Cahn-Hilliard model based on the ternary model (8) introduced and studied in the beginning of this article. The coupling we propose is classical and lets us take into account hydrodynamics of ternary systems and in particular, capillary effects at interfaces.

Then, after a brief description of the numerical schemes we used, we present the results obtained for the classical test case of a lens located at the interface between the other two components. We discuss with these results the validity of our model and we illustrate the fact that the properties of algebraic and dynamical consistency with binary systems, that we studied in previous sections, are crucial for this kind of models.

Finally, the full model is validated through the computation of a bubble rising across a liquid/liquid interface. We obtain in this case good agreement with theoretical predictions.

\subsection{Navier-Stokes/Cahn-Hilliard system}

The previous diffuse interface model can be easily coupled with the Navier-Stokes equations in order to take into account the quasi-incompressible hydrodynamics of the mixture. This coupling (but also some variants) can be justified by several means that we do not develop here (see $[6,15,19,21])$.

The velocity jump being zero between two phases, we can define an unique mixture mean velocity $u$, smooth on the domain. Thus, in order to couple the equations, we add a convection term in the evolution equation for the concentrations as follows

$$
\frac{\partial c_{i}}{\partial t}+(u \cdot \nabla) c_{i}=\nabla \cdot\left(\frac{M_{0}}{\Sigma_{i}} \nabla \mu_{i}\right),
$$

the definition of the chemical potential $\mu_{i}$ being the same as in (8). Then, the evolution of the mean velocity $u$ is described by the non-homogeneous Navier-Stokes equation,

$$
\left\{\begin{array}{l}
\varrho\left(\frac{\partial u}{\partial t}+u \cdot \nabla u\right)-\nabla \cdot(2 \eta D(u))+\nabla p=\sum_{i=1}^{3} \mu_{i} \nabla c_{i}+\varrho g \\
\nabla \cdot u=0
\end{array}\right.
$$

where $D(u)=\left(\nabla u+{ }^{t} \nabla u\right) / 2$ is the symmetric part of the gradient of $u$ and the thermophysical properties, such that the density and the dynamical viscosity, respectively denoted by $\varrho_{i}$ and $\eta_{i}$ in the phase $i$, are approximated by smooth functions, which depend on the order parameters and satisfy

$$
\varrho\left(c_{1}, c_{2}, c_{3}\right)=\varrho_{i} \text { if } c_{i}=1 \quad \text { and } \quad \eta\left(c_{1}, c_{2}, c_{3}\right)=\eta_{i} \text { if } c_{i}=1 .
$$

The first source term in this equation represents the capillary forces which takes place at each of the interfaces between the three components. Notice that, if we try to write the evolution of the total energy of the system (kinetic energy + Cahn-Hilliard energy) then we have to multiply the evolution equation for each $c_{i}$ by $\mu_{i}$ and the Navier-Stokes equation by $u$ and then we sum up the results. We see that the contribution of all the convection terms in the Cahn-Hilliard system is exactly balanced with the contribution of the capillary forces. 


\subsection{Numerical schemes}

In order to solve the problem, we use a Galerkin finite elements method. The velocity is discretized using the classical $\mathbb{P}_{1}$ bubble element whereas the pressure, the order parameters and the chemical potentials are approximated using the $\mathbb{P}_{1}$ element. Since the pressure and the order parameters are chosen in the same approximation space, we see that the contribution of convection terms in the evolution of the total volume of each constituent is exactly zero.

The temporal interval is uniformly discretized, with a fixed time step $\Delta t$. A semi-discretization in time is used to decouple the Cahn-Hilliard and Navier-Stokes equations in a time step. In order to do this, the Cahn-Hilliard equations are first solved with an explicit advection velocity

$$
\left\{\begin{aligned}
\frac{c_{i}^{n+1}-c_{i}^{n}}{\Delta t}+\left(u^{n} \cdot \nabla\right) c_{i}^{n+1} & =\nabla \cdot\left(\frac{M_{0}\left(\mathbf{C}^{n}\right)}{\Sigma_{i}} \nabla \mu_{i}^{n+1}\right), \\
\mu_{i}^{n+1} & =-\frac{3}{4} \varepsilon \Sigma_{i} \Delta c_{i}^{n+1}+\frac{4 \Sigma_{T}}{\varepsilon} \sum_{j \neq i} \frac{1}{\Sigma_{j}}\left(\partial_{i} F\left(\mathbf{C}^{n+1}\right)-\partial_{j} F\left(\mathbf{C}^{n+1}\right)\right),
\end{aligned}\right.
$$

for each $i \in\{1,2\}$. Notice that we allow the mobility $M_{0}$ to depend on $\mathbf{C}$ even though our analysis in this paper only concerns a constant mobility coefficient. The resolution is performed using a Newton method, with an explicit mobility. In the second step, the Navier-Stokes equations are solved using a standard augmented Lagrangian technique or a penalty-projection method [17]. We use the concentrations and the chemical potentials computed from the first step to define capillary forces. Moreover, the advection term is linearized using an explicit advection velocity. Noting $\varrho^{n+1}=\varrho\left(\mathbf{C}^{n+1}\right)$ and $\eta^{n+1}=\eta\left(\mathbf{C}^{n+1}\right)$, the Navier-Stokes equation are written

$$
\left\{\begin{array}{l}
\varrho^{n+1}\left(\frac{u^{n+1}-u^{n}}{\Delta t}+\left(u^{n} \cdot \nabla\right) u^{n+1}\right)-\nabla \cdot\left(2 \eta^{n+1} D\left(u^{n+1}\right)\right)+\nabla p^{n+1}=\sum_{i=1}^{3} \mu_{i}^{n+1} \nabla c_{i}^{n+1}+\varrho^{n+1} g \\
\nabla \cdot u^{n+1}=0 .
\end{array}\right.
$$

The practical implementation has been performed using the software object-oriented component library PELICANS, developed at IRSN (see [24]).

\subsection{Liquid lens between two stratified fluids}

We begin the illustration of the behavior of our model by the classical test case of the liquid lens which is initially spherical and located at the interface between two other immiscible fluids (see [26] for instance). We study the lens spreading which can be partial or total (see $[25]$ ). The domain is $\Omega=] 0.0,0.1[\times] 0.02,0.08[$ and the triangular mesh has approximately 40800 elements (spatial step $h=8.33 \times 10^{-4}$ ). The interface width is taken to be $\varepsilon \sim 3 h$. The mobility is taken constant or degenerate. Even though non-degenerate and degenerate models lead to sharp interface limits with different time scales (see $[1,11]$ ), both solutions are identical at the equilibrium.

\subsubsection{Partial spreading}

When all coefficients $\left(\Sigma_{i}\right)_{i}$ are positive, the lens spreading is said to be partial. In that case, the equilibrium state in the limit $\varepsilon \rightarrow 0$ can be computed analytically: the final shape of the lens is the union of two pieces of circles, the contact angles being given as a function of the three surface tensions by the Young relations as shown in Figure 3.

We solve here the full Navier-Stokes/Cahn-Hilliard system. The time step $\Delta t$ is equal to 0.001 and the mobility is taken degenerate $\left(M_{0}=5 \cdot 10^{-7}\left(1-c_{1}\right)^{2}\left(1-c_{2}\right)^{2}\left(1-c_{3}\right)^{2}\right)$. We give below in Figure 4 the results obtained with the bulk free energy $F_{0}$ (which is a reasonable choice here since all the $\Sigma_{i}$ are positive in the presented situations). The white zone corresponds to the points where $\left(1-c_{1}\right)\left(1-c_{2}\right)\left(1-c_{3}\right)>\frac{3}{16}$ 


$$
\frac{\sin \theta_{1}}{\sigma_{23}}=\frac{\sin \theta_{2}}{\sigma_{13}}=\frac{\sin \theta_{3}}{\sigma_{12}}
$$

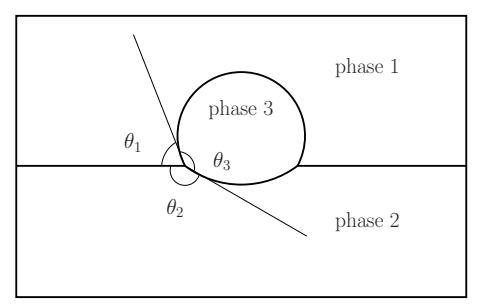

FIGURE 3. Theoretical shape of the lens at the equilibrium.

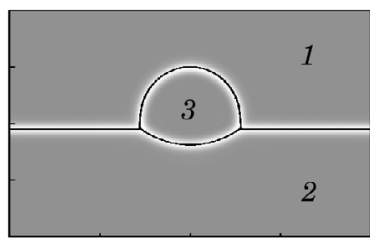

$(1 ; 0.8 ; 1.4)$

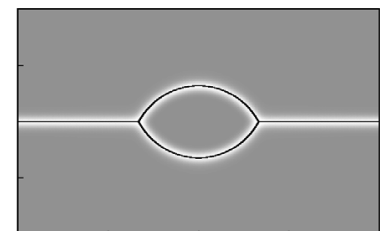

$(1 ; 1 ; 1)$

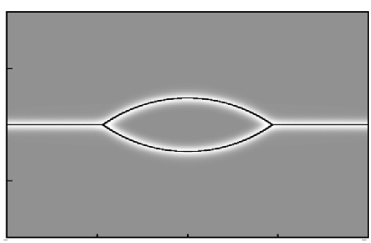

$(1 ; 0.6 ; 0.6)$

Figure 4. Equilibrium states obtained with $F=F_{0}$ for different surface tensions $\left(\sigma_{12} ; \sigma_{13} ; \sigma_{23}\right)$.

TABLE 1. Relative error for the pressure jump at $t=7.5$.

\begin{tabular}{|c|c|c|c|c|c|}
\hline surface tensions & theoretical pressure jump & \multicolumn{4}{|c|}{ relative error } \\
\cline { 3 - 6 }$\left(\sigma_{12} ; \sigma_{13} ; \sigma_{23}\right)$ & $p_{3}-p_{2}=p_{3}-p_{1}$ & $h=1.4 \times 10^{-3}$ & $h=10^{-3}$ & $h=8.3 \times 10^{-4}$ & $h=7 \times 10^{-4}$ \\
\hline$(1 ; 0.8 ; 1.4)$ & 57.87 & $3.1 \%$ & $2.3 \%$ & $2 \%$ & $1.9 \%$ \\
$(1 ; 1 ; 1)$ & 52.11 & $3.05 \%$ & $2.1 \%$ & $1.8 \%$ & $1.7 \%$ \\
$(1 ; 0.6 ; 0.6)$ & 14.11 & $3.9 \%$ & $2.7 \%$ & $2.6 \%$ & $2.5 \%$ \\
\hline
\end{tabular}

(which can be considered as the approximate interfacial zone) and the black solid line stands for the location of the interface in the analytic solution of the problem. We remark that the shape of the lens and the contact angles at triple point are correctly captured even though the interface thickness $\varepsilon$ is not zero.

Another usual way to validate our model is the Laplace law which says that, at the equilibrium in the sharp interface limit $\varepsilon \rightarrow 0$, the pressures $p_{1}, p_{2}$ in the phases 1 and 2 are equal and that the pressure $p_{3}$ in the lens satisfies

$$
\frac{\sigma_{13}}{r_{13}}=p_{3}-p_{1}=p_{3}-p_{2}=\frac{\sigma_{23}}{r_{23}}
$$

where $r_{i 3}$ is the radius of the circle being at the interface between the phase $i$ and the lens. The interface thickness is fixed at 0.0028 and the spatial step $h$ varies. We see in Table 1 that our model give satisfactory results for the pressure jumps across the diffuse interfaces.

\subsubsection{Comparisons with non consistent models}

We compare in this section our algebraically consistent model with $F=F_{0}$ with the non-consistent one based on $\widetilde{F}_{0}$ and with the model studied in $[18,20]$, which is also based on $\widetilde{F}_{0}$ but is built in a different way than what we do in Section 2.1.

We show in Figure 5 the results obtained for these three models on the test case of the lens where only Cahn-Hilliard equations are solved. The mobility is constant and $\Delta t=1$. We see that only the algebraically consistent model gives correct results across the interfaces since for the other models, the phase $i$ appears in the middle of the interface between the other two phases $j$ and $k$, which is of course not physical. As shown in our 

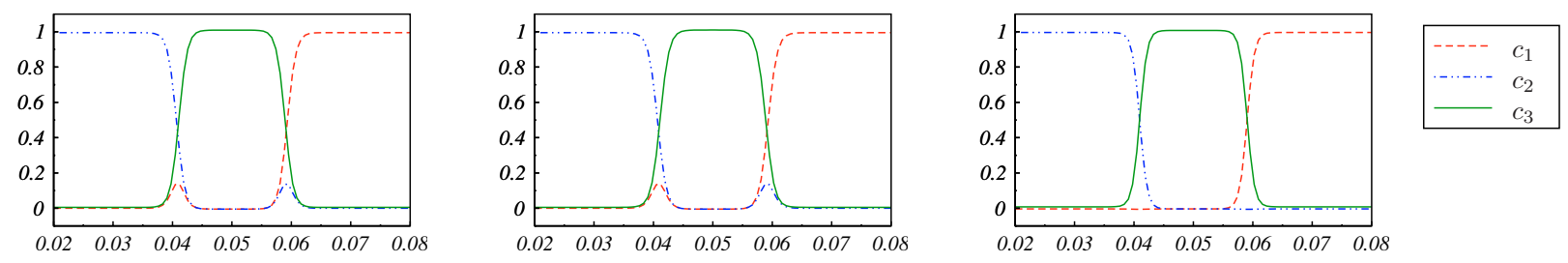

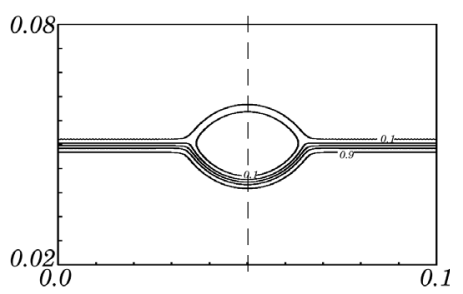

Non-consistent case model proposed in [20]

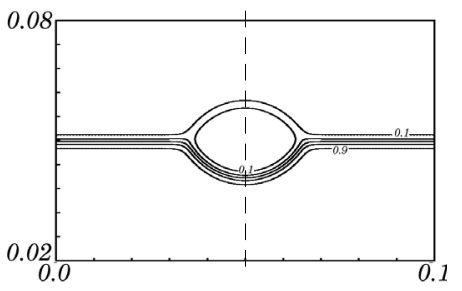

Non-consistent case our model with $F=\widetilde{F}_{0}$

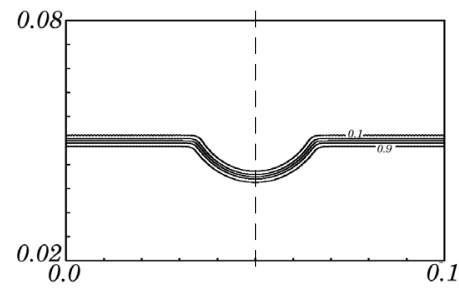

Consistent case our model with $F=F_{0}$

Figure 5. Vertical cutline of order parameters at $x=0.05$ for $\left(\sigma_{12} ; \sigma_{13} ; \sigma_{23}\right)=(1 ; 1 ; 1)$ and contour line of $c_{2}$ at 5 levels $c_{2}=0.1,0.3,0.5,0.7,0.9$ for three different models.

analytical study of previous sections this phenomenon is closely linked to the structure of the model and then persists under refinement of the mesh or of the interfacial width $\varepsilon$. Notice that these fact was already observed in ([20], Fig. 4.5).

Hence, the use of our new model for the bulk free energy is crucial and gives very significant qualitative improvement of the results compared to the various models found in the literature. The consistency with diphasic systems (see Thms. 3.2, 3.8 and 3.9) prevents the model from non-physical apparition of one of the component in the interface between the other two.

\subsubsection{Non-dynamically consistent models}

The importance of the dynamical consistency of the model is also illustrated in this section. An example is given where the condition (48)

$$
\Sigma_{i}>\frac{\Sigma_{T}}{2}>0
$$

of Theorem 3.9 is not satisfied. Like in Theorem $3.9, F$ is equal to $F_{0}$ and the mobility is chosen to be constant. Surface tensions are $\left(\sigma_{12} ; \sigma_{13} ; \sigma_{23}\right)=(1 ; 0.5 ; 0.55)$ so that we have

$$
\Sigma_{3}=0.05<\frac{\Sigma_{T}}{2} \simeq 0.068
$$

We only solve the Cahn-Hilliard equations and choose $\Delta t=1$.

Hence, Theorem 3.9 does not hold and we do not know if the model is dynamically consistent. Nevertheless, we observe in Figure 6 that there is artificial apparition of the phase represented by $c_{3}$ in the interface between the two stratified phases. Notice that this apparition is much less important than in Figure 5. This is explained by the fact that the problem here does not come from a structural defect of the model (since it is algebraically consistent) but only from a dynamical instability which develops due to numerical errors.

If now we take $F$ to be $F_{\Lambda, 0}$ for $\Lambda$ large enough, Theorem 3.8 holds. In this case, the model is dynamically consistent and the numerical instabilities almost disappear as shown in Figure 6. 

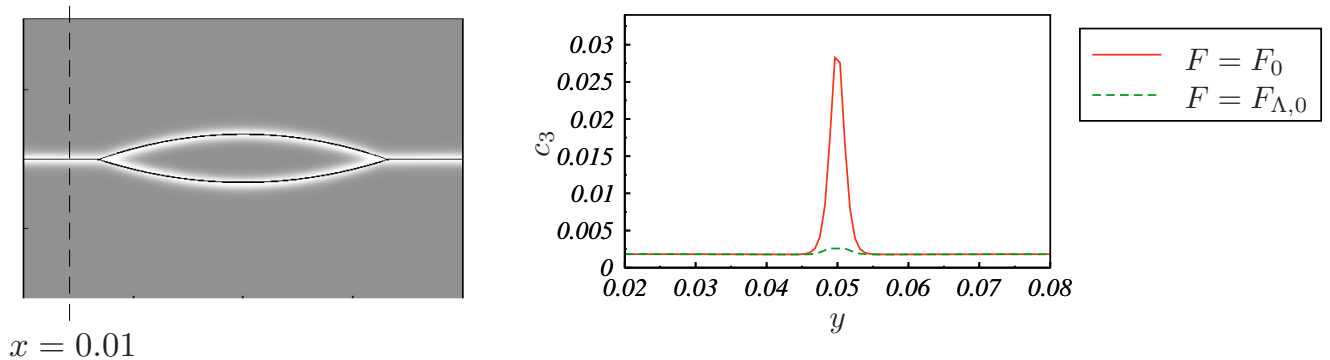

Figure 6 . Equilibrium state obtained for $\left(\sigma_{12} ; \sigma_{13} ; \sigma_{23}\right)=(1 ; 0.5 ; 0.55)$ and vertical cutline of the order parameter $c_{3}$ at $x=0.01$ for $F=F_{0}$ and $F=F_{\Lambda, 0}$ with $\Lambda=7$.

$\left(\sigma_{12} ; \sigma_{13} ; \sigma_{23}\right)=(3 ; 1 ; 1), \Sigma_{3}<0$
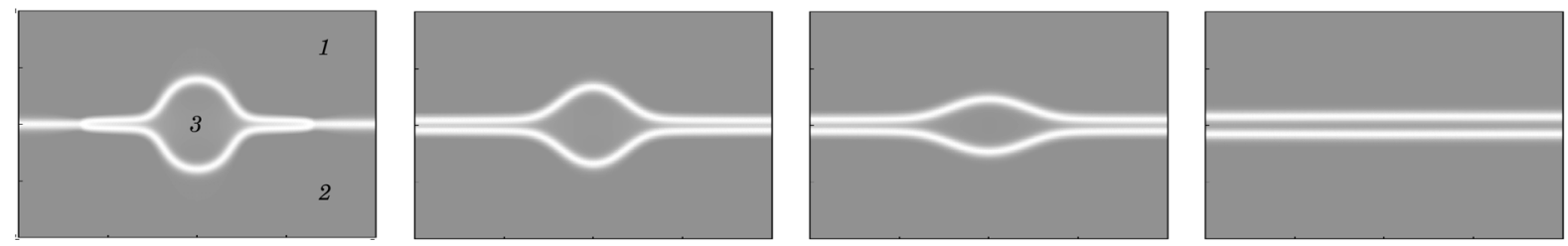

$$
\left(\sigma_{12} ; \sigma_{13} ; \sigma_{23}\right)=(1 ; 1 ; 3), \Sigma_{1}<0
$$
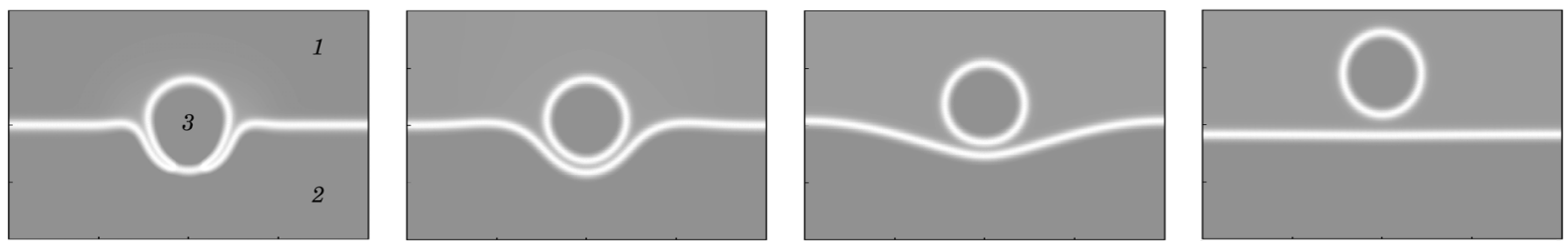

Figure 7 . Evolution in time of the lens in total spreading cases, using $F=F_{\Lambda, 0}$ with $\Lambda=7$.

\subsubsection{Total spreading}

In the case where one of coefficients $\Sigma_{i}$ is negative, the spreading is said to be total. We use here the bulk free energy $F_{\Lambda, 0}$ with $\Lambda=7$ which ensures stability of the model. We only solve the Cahn-Hilliard equations with constant mobility and $\Delta t=0.02$ since we are not interested in this test case with hydrodynamics. Two configurations are shown in Figure 7 where the surface tensions are respectively $(3 ; 1 ; 1)$ and $(1 ; 1 ; 3)$. We can see that in both cases, the phase $i$ associated to the negative coefficient $\Sigma_{i}$, spreads between the other two.

Once again, it is important to notice that the use of the high order bulk free energy $F_{\Lambda, 0}$ with $\Lambda$ large enough is fundamental here. Indeed, if we try to use $F=F_{0}$ in this test case then the bulk free energy takes negative values and then the computation rapidly blows up because the concentrations do not remain bounded. We compare in Figure 8 the results obtained at the same time (after a few time steps and before the blow up) in the two cases $F=F_{0}$ and $F=F_{\Lambda, 0}$. 

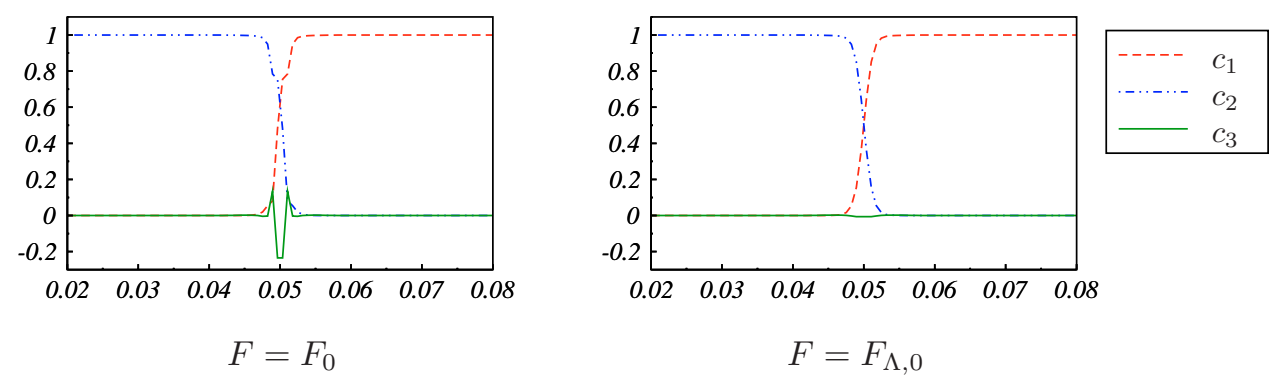

Figure 8. Vertical cutline of the order parameters at $x=0.035$ for $\left(\sigma_{12} ; \sigma_{13} ; \sigma_{23}\right)=(3 ; 1 ; 1)$.

TABle 2. Physical properties.

\begin{tabular}{|c|c|c|}
\hline & $\varrho\left(\mathrm{kg} \cdot \mathrm{m}^{-3}\right)$ & $\eta$ (Pa.s) \\
\hline bubble $\left(c_{1}\right)$ & 1 & $10^{-4}$ \\
light liquid $\left(c_{2}\right)$ & 1000 & $10^{-1}$ \\
heavy liquid $\left(c_{3}\right)$ & 1500 & $1.510^{-1}$ \\
\hline
\end{tabular}

\begin{tabular}{|c|c|}
\hline \multicolumn{2}{|c|}{ surface tensions (N.m $\left.{ }^{-1}\right)$} \\
\hline gaz/liquid $\sigma_{12}, \sigma_{13}$ & 0.07 \\
liquid/liquid $\sigma_{23}$ & 0.1 \\
\hline
\end{tabular}

TABLE 3. Results obtained for the bubble penetration for different radii on the mesh with 34400 elements.

\begin{tabular}{|c|c|c|c|c|c|c|}
\hline radius $r(\mathrm{~m})$ & 0.0022 & 0.0032 & 0.0034 & 0.0035 & 0.0039 & 0.0042 \\
\hline bubble penetration & no & no & no & yes & yes & yes \\
\hline
\end{tabular}

\subsection{Rising bubbles}

In this final section, we study the bubble rising across a liquid/liquid interface. Two behaviors can occur: the bubble can penetrate the liquid/liquid interface and rise into the upper liquid layer or it can remain captured between the two liquid layers. Greene et al. [14] propose a criterion on the bubble's volume to predict the penetration in the light fluid. The criterion only takes into account Archimede's force and surface tension forces. They predict that the bubble crosses the interface if its volume $V$ is greater than

$$
V^{*}=\left[\frac{2 \pi\left(\frac{3}{4 \pi}\right)^{\frac{1}{3}} \sigma_{23}}{g\left(\varrho_{2}-\varrho_{1}\right)}\right]^{\frac{3}{2}} .
$$

The physical parameters we chose are detailed in Table 2 and we get that $V^{*} \simeq 2.5 \times 10^{-7}$ which leads to a critical radius $r^{*} \simeq 0.0039$. We assume here that the flow is $3 \mathrm{D}$ axisymmetric and that the $2 \mathrm{D}$ computational domain $[0 ; 2 r] \times[0 ; 15 r], r$ being the radius of the bubble. The triangular mesh has approximately 34400 elements and the interface width $\varepsilon=0.15 r$ is chosen so that approximately 3 triangles of the mesh lie in the interfaces. The time step is $0.001 \mathrm{~s}$. The two behaviors are obtained as shown in Figure 9 and we find that there exists a numerical critical radius $r_{\text {num }}^{*} \sim 0.0035$ as reported in Table 3 . In Table 4 , we can see that the value of $r_{\text {num }}^{*}$ tends to 0.0036 when the mesh is refined with $\varepsilon=0.15 r$ fixed.

Notice that it is coherent that $r_{\text {num }}^{*}<r^{*}$ because the theoretical criterion given in [14] overestimates the required force for the bubble crossing and neglects many hydrodynamics effects. 

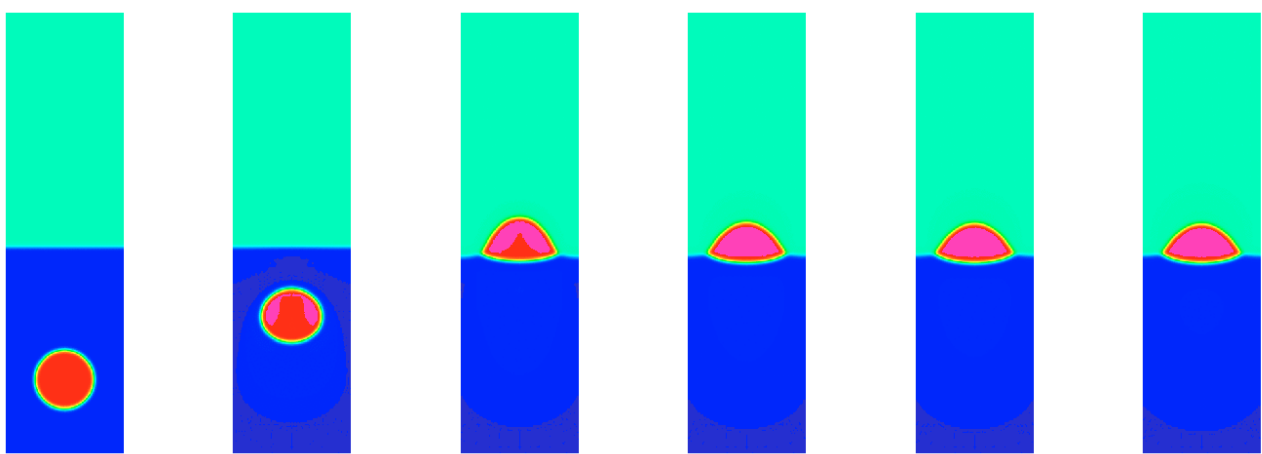

$r=0.0042>r_{\text {num }}^{*}$

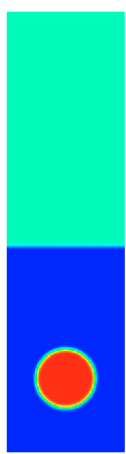

$t=0$

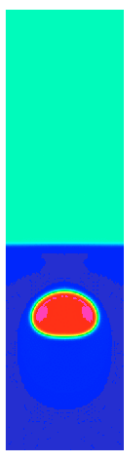

$t=0.067$

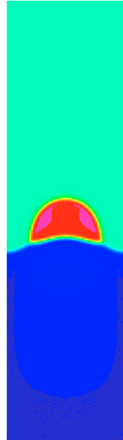

$t=0.16$

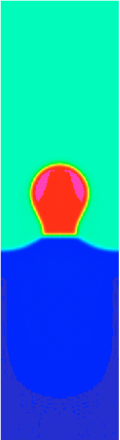

$t=0.2$

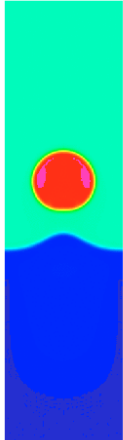

$t=0.214$

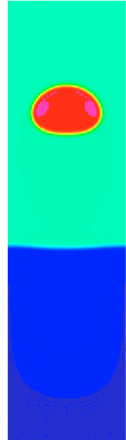

$t=0.28$

Figure 9. Bubble rising across a liquid/liquid interface for $r=0.0032$ and $r=0.0042$.

TABLE 4. Values of $r_{\text {num }}^{*}$ for different meshes with $\varepsilon$ fixed.

\begin{tabular}{|l|c|c|c|c|}
\hline number of cells & 19320 & 34400 & 53400 & 76800 \\
\hline radius $r_{\text {num }}^{*}(\mathrm{~m})$ & 0.0034 & 0.0035 & 0.0036 & 0.0036 \\
\hline
\end{tabular}

\section{Conclusion}

In this article we proposed the construction and the analysis of particular ternary Cahn-Hilliard models for which well-posedness and consistency properties are ensured. We pay particular attention to the total spreading cases and our analysis is valid in all the cases where condition (11) holds. We also describe the coupling of the ternary Cahn-Hilliard model with the Navier-Stokes equations by taking into account capillary effects on the hydrodynamics of the mixture. Finally, we give numerical results which illustrate our analysis but also the behavior and the potentiality of the model.

To our knowledge, there are still open questions in the field. For instance, is it possible to propose a ternary Cahn-Hilliard model which leads to satisfactory results even for total spreading cases which do not satisfy condition (11), or in cases where the surface tensions are not constant (if one wishes to model impurities for instance)?

One can also wonder if it is possible to generalize our study to more than three components. Of course, the general description of multi-component systems described in Section 2.1 is valid for any number of components (see for instance $[3,10,13]$ ) and can be useful if one is interested in systems with phase changes. 
Nevertheless, the use of such models for more than three immiscible components is not straightforward and we do not know if an analysis similar to the one we propose in this paper is possible to ensure consistency properties in such cases.

\section{A. Proofs of Propositions 3.6 And 3.7}

Proof of Proposition 3.6. Suppose for instance that $\Sigma_{1}<0$. Since all the $\sigma_{i j}$ are positive, we deduce from (22) that necessarily $\Sigma_{2}>0$ and $\Sigma_{3}>0$.

$\mathbf{1}$ / Using (34), we see that for any $\mathbf{C} \in \mathcal{S}$ we have

$$
\begin{aligned}
F_{\Lambda, \alpha}(\mathbf{C}) & \geq-\frac{\left|\Sigma_{1}\right|}{2} c_{1}^{2}\left(c_{2}+c_{3}\right)^{2}+\Lambda c_{1}^{2} c_{2}^{2} c_{3}^{2}\left(\varphi_{\alpha}\left(c_{1}\right)+\varphi_{\alpha}\left(c_{2}\right)+\varphi_{\alpha}\left(c_{3}\right)\right) \\
& \geq-\frac{\left|\Sigma_{1}\right|}{2} c_{1}^{2}\left(c_{2}+c_{3}\right)^{2}+\Lambda c_{1}^{2}\left(\Phi_{\alpha}\left(c_{2}\right) c_{3}^{2}+\Phi_{\alpha}\left(c_{3}\right) c_{2}^{2}\right) .
\end{aligned}
$$

Let us define

$$
M_{\Lambda, \alpha}=\Phi_{\alpha}^{-1}\left(\frac{\left|\Sigma_{1}\right|}{\Lambda}\right) .
$$

For any $\mathbf{C} \in \mathcal{S}$ such that $\left|c_{2}\right| \geq M_{\Lambda, \alpha}$ and $\left|c_{3}\right| \geq M_{\Lambda, \alpha}$, we have

$$
\begin{aligned}
F_{\Lambda, \alpha}(\mathbf{C}) & \geq-\frac{\left|\Sigma_{1}\right|}{2} c_{1}^{2}\left(c_{2}+c_{3}\right)^{2}+\Lambda \Phi_{\alpha}\left(M_{\Lambda, \alpha}\right) c_{1}^{2}\left(c_{3}^{2}+c_{2}^{2}\right) \\
& \geq-\frac{\left|\Sigma_{1}\right|}{2} c_{1}^{2}\left(c_{2}+c_{3}\right)^{2}+\frac{\Lambda}{2} \Phi_{\alpha}\left(M_{\Lambda, \alpha}\right) c_{1}^{2}\left(c_{2}+c_{3}\right)^{2}=0,
\end{aligned}
$$

by definition of $M_{\Lambda, \alpha}$. Hence, we have shown that $F_{\Lambda, \alpha}(\mathbf{C})$ is non-negative as soon as $\mathbf{C} \in \mathcal{S}$ is such that $\left|c_{2}\right| \geq M_{\Lambda, \alpha}$ and $\left|c_{3}\right| \geq M_{\Lambda, \alpha}$.

2/ Let us now consider $\mathbf{C} \in \mathcal{S}$ such that $\left|c_{2}\right| \leq M_{\Lambda, \alpha}$, for instance. The same study can be done in the case $\left|c_{3}\right| \leq M_{\Lambda, \alpha}$. Using (35) and since $\Lambda>0$ and $\Sigma_{2}>0$, we have for such a $\mathbf{C}$ :

$$
F_{\Lambda, \alpha}(\mathbf{C}) \geq \frac{\Sigma_{1}}{2} c_{1}^{2}\left(1-c_{1}\right)^{2}+\frac{\Sigma_{3}}{2}\left(1-c_{1}-c_{2}\right)^{2}\left(c_{1}+c_{2}\right)^{2} .
$$

If we denote by $G\left(c_{1}, c_{2}\right)$ the right-hand side member of this inequality, we can write

$$
G\left(c_{1}, c_{2}\right)=\frac{\Sigma_{1}}{2} c_{1}^{2}\left(1-c_{1}\right)^{2}+\frac{\Sigma_{3}}{2} c_{1}^{2}\left(1-c_{1}\right)^{2}+\frac{\Sigma_{3}}{2} H\left(c_{1}, c_{2}\right),
$$

with

$$
H\left(c_{1}, c_{2}\right)=c_{2}\left(\left(1-c_{1}\right)^{2}\left(2 c_{1}+c_{2}\right)-2\left(1-c_{1}\right)\left(c_{1}+c_{2}\right)^{2}+c_{2}\left(c_{1}+c_{2}\right)^{2}\right) .
$$

Using that $\left|c_{2}\right|$ is assumed to be bounded by $M_{\Lambda, \alpha}$, one obtains

$$
\left|\frac{\Sigma_{3}}{2} H\left(c_{1}, c_{2}\right)\right| \leq K M_{\Lambda, \alpha}\left(1+M_{\Lambda, \alpha}^{3}\right)\left(1+\left|c_{1}\right|^{3}\right)
$$

where $K$ depend only on $\left|\Sigma_{3}\right|$. Using Young's inequality, it follows that for all $0<\delta<1$

$$
\left|\frac{\Sigma_{3}}{2} H\left(c_{1}, c_{2}\right)\right| \leq K M_{\Lambda, \alpha}\left(1+M_{\Lambda, \alpha}^{3}\right)+\frac{\delta \sigma_{13}}{2} c_{1}^{4}+\frac{K}{\delta^{3}} M_{\Lambda, \alpha}^{4}\left(1+M_{\Lambda, \alpha}^{12}\right) .
$$

Then, we use the following elementary result whose proof is left to the reader. 
Lemma A.1. For all $0<\delta<1$, we have

$$
x^{2}(1-x)^{2} \geq \frac{\delta}{2} x^{4}-\frac{1}{2} \frac{\delta}{(1-\delta)^{2}}, \quad \forall x \in \mathbb{R} .
$$

It follows from (76) that

$$
\begin{aligned}
G\left(c_{1}, c_{2}\right) & \geq \sigma_{13} c_{1}^{2}\left(1-c_{1}\right)^{2}-\left|\frac{\Sigma_{3}}{2} H\left(c_{1}, c_{2}\right)\right| \\
& \geq-K M_{\Lambda, \alpha}\left(1+M_{\Lambda, \alpha}^{3}\right)-\frac{K}{\delta^{3}} M_{\Lambda, \alpha}^{4}\left(1+M_{\Lambda, \alpha}^{12}\right)-\frac{1}{2} \frac{\delta \sigma_{13}}{(1-\delta)^{2}}
\end{aligned}
$$

which proves that $G$ (and so also $F_{\Lambda, \alpha}$ ) is bounded from below for such values of $\mathbf{C}$. The first part of the proposition is proved.

Finally, for $\Lambda$ large enough, we can see that $M_{\Lambda, \alpha}$ is smaller than 1 , so we can take $\delta=M_{\Lambda, \alpha}$ in the previous inequality which implies that

$$
\inf _{\mathcal{S}} F_{\Lambda, \alpha}(\mathbf{C}) \geq-K M_{\Lambda, \alpha}\left(1+M_{\Lambda, \alpha}^{3}\right)-K M_{\Lambda, \alpha}\left(1+M_{\Lambda, \alpha}^{12}\right)-\frac{1}{2} \frac{M_{\Lambda, \alpha} \sigma_{13}}{\left(1-M_{\Lambda, \alpha}\right)^{2}}
$$

The claim is proved because the right hand-side of the inequality above tends to zero when $\Lambda$ tends to $\infty$, since we have $\lim _{\Lambda \rightarrow+\infty} M_{\Lambda, \alpha}=0$.

Proof of Proposition 3.7. First of all, if condition (46) is not satisfied then $\inf _{\mathcal{S}} F_{\Lambda, \alpha}<0$. Indeed, in that case there exists $(a, b) \neq(0,0)$ such that (see the Proof of Prop. 2.1)

$$
\Sigma_{1} a^{2}+\Sigma_{2} b^{2}+\Sigma_{3}(a+b)^{2}<0
$$

It is easily seen in that case that, for any $\alpha$ and any $\Lambda$, we have

$$
F_{\Lambda, \alpha}(a x, b x, 1-(a x+b x)) \underset{x \rightarrow 0}{\sim}\left(\Sigma_{1} a^{2}+\Sigma_{2} b^{2}+\Sigma_{3}(a+b)^{2}\right) x^{2}
$$

so that $F_{\Lambda, \alpha}$ takes negative values on $\mathcal{S}$ in the neighborhood of $(0,0,1)$. Similar arguments show that $F_{\Lambda, \alpha}$ takes negative values in the case $\Sigma_{1} \Sigma_{2}+\Sigma_{1} \Sigma_{3}+\Sigma_{2} \Sigma_{3}=0$.

Assume now that (46) holds. We want to show that the function $H_{\Lambda, \alpha}$ defined by

$$
H_{\Lambda, \alpha}\left(c_{1}, c_{2}\right)=F_{\Lambda, \alpha}\left(c_{1}, c_{2}, 1-c_{1}-c_{2}\right)
$$

is non-negative for $\Lambda$ large enough. Obviously, for any $\left(c_{1}, c_{2}\right)$ we can not have simultaneously

$$
\left|c_{1}\right|<\frac{1}{4},\left|c_{2}\right|<\frac{1}{4},\left|1-c_{1}-c_{2}\right|<\frac{1}{4}
$$

Assume for instance that $\left|1-c_{1}-c_{2}\right| \geq \frac{1}{4}$, the other two cases can be treated in the same way. Using Corollary 2.2 and (35), (37), we have

$$
\begin{aligned}
H_{\Lambda, \alpha}\left(c_{1}, c_{2}\right)= & \frac{1}{2} \Sigma_{1}\left[c_{1}\left(1-c_{1}\right)\right]^{2}+\frac{1}{2} \Sigma_{2}\left[c_{2}\left(1-c_{2}\right)\right]^{2}+\frac{1}{2} \Sigma_{3}\left[\left(c_{1}+c_{2}\right)\left(1-c_{1}-c_{2}\right)\right]^{2} \\
& +\Lambda c_{1}^{2} c_{2}^{2}\left(1-c_{1}-c_{2}\right)^{2}\left(\varphi_{\alpha}\left(c_{1}\right)+\varphi_{\alpha}\left(c_{2}\right)+\varphi_{\alpha}\left(1-c_{1}-c_{2}\right)\right) \\
\geq & -\frac{1}{2} \bar{\Sigma}\left[c_{1}\left(1-c_{1}\right)+c_{2}\left(1-c_{2}\right)-\left(c_{1}+c_{2}\right)\left(1-c_{1}-c_{2}\right)\right]^{2}+\Lambda c_{1}^{2} c_{2}^{2} \Phi_{\alpha}\left(1-c_{1}-c_{2}\right) .
\end{aligned}
$$


A straightforward computation shows that the first term equals $-\frac{1}{2} \bar{\Sigma}\left(2 c_{1} c_{2}\right)^{2}$. Furthermore, since we assumed that $\left|1-c_{1}-c_{2}\right| \geq \frac{1}{4}$ and thanks to (39) we have $\Phi_{\alpha}\left(1-c_{1}-c_{2}\right) \geq \Phi_{\alpha}\left(\frac{1}{4}\right)$. Hence it follows that

$$
H_{\Lambda, \alpha}\left(c_{1}, c_{2}\right) \geq-2 \bar{\Sigma} c_{1}^{2} c_{2}^{2}+\Lambda c_{1}^{2} c_{2}^{2} \Phi_{\alpha}\left(\frac{1}{4}\right)
$$

and this last quantity is non-negative for any $c_{1}, c_{2}$ as soon as

$$
\Lambda \geq \frac{2 \bar{\Sigma}}{\Phi_{\alpha}\left(\frac{1}{4}\right)} .
$$

Acknowledgements. The authors want to express their gratitude to B. Piar for his support during the preparation of this work and to the referees for their careful reading of this paper. They also want to thank P. Angot, J.C. Latché and M. Quintard for their interest in these researches.

The work of the second author has been partly supported by the Région Provence-Alpes-Côte d'Azur and followed by the OPTIFLOW company.

\section{REFERENCES}

[1] N.D. Alikakos, P.W. Bates and X. Chen, Convergence of the Cahn-Hilliard equation to the Hele-Shaw model. Arch. Ration. Mech. An. 128 (1994) 165-205.

[2] D.M. Anderson, G.B. McFadden and A.A. Wheeler, Diffuse-interface methods in fluid mechanics. Annu. Rev. Fluid Mech. 30 (1998) 139-165.

[3] J.W. Barrett and J.F. Blowey, Finite element approximation of a model for phase separation of a multi-component alloy with non-smooth free energy and a concentration dependent mobility matrix. Math. Mod. Meth. Appl. S. 9 (1999) 627-663.

[4] J.F. Blowey, M.I.M. Copetti and C.M. Elliott, Numerical analysis of a model for phase separation of a multi-component alloy. IMA J. Numer. Anal. 16 (1996) 111-139.

[5] F. Boyer, Mathematical study of multiphase flow under shear through order parameter formulation. Asymptotic Anal. 20 (1999) $175-212$.

[6] F. Boyer, A theoretical and numerical model for the study of incompressible mixture flows. Comput. Fluids 31 (2002) 41-68.

[7] M.I.M. Copetti, Numerical experiments of phase separation in ternary mixtures. Math. Comput. Simulat. 52 (2000) 41-51.

[8] C.M. Elliott, The Cahn-Hilliard model for the kinetics of phase separation, in Mathematical Models for Phase Change Problems, J.F. Rodrigues Ed., Birkhäuser Verlag Basel. Intern. Ser. Numer. Math. 88 (1989).

[9] C.M. Elliott and S. Luckhaus, A generalised diffusion equation for phase separation of a multi-component mixture with interfacial free energy. IMA Preprint Series 887 (1991).

[10] D.J. Eyre, Systems of Cahn-Hilliard equations. SIAM J. Appl. Math. 53 (1993) 1686-1712.

[11] H. Garcke and A. Novick-Cohen, A singular limit for a system of degenerate Cahn-Hilliard equations. Adv. Differ. Equ. 5 (2000) 401-434.

[12] H. Garcke, B. Nestler and B. Stoth, On anisotropic order parameter models for multi-phase systems and their sharp interface limits. Physica D 115 (1998) 87-108.

[13] H. Garcke, B. Nestler and B. Stoth, A multi phase field: numerical simulations of moving phase boundaries and multiple junctions. SIAM J. Appl. Math. 60 (1999) 295-315.

[14] G.A. Greene, J.C. Chen and M.T. Conlin, Onset of entrainment between immiscible liquid layers due to rising gas bubbles. Int. J. Heat Mass Tran. 31 (1988) 1309-1317.

[15] D. Jacqmin, Calculation of two-phase Navier-Stokes flows using phase-field modeling. J. Comput. Phys. 155 (1999) $96-127$.

[16] D. Jacqmin, Contact-line dynamics of a diffuse fluid interface. J. Fluid Mechanics 402 (2000) 57-88.

[17] M. Jobelin, C. Lapuerta, J.-C. Latché, P. Angot and B. Piar, A finite element penalty-projection method for incompressible flows. J. Comput. Phys. (2006) (to appear).

[18] J. Kim, Modeling and simulation of multi-component, multi-phase fluid flows. Ph.D. thesis, Univeristy of California, Irvine (2002).

[19] J. Kim, A continuous surface tension force formulation for diffuse-interface models. J. Comput. Phys. 204 (2005) 784-804.

[20] J. Kim and J. Lowengrub, Phase field modeling and simulation of three-phase flows. Interfaces and Free Boundaries 7 (2005) 435-466.

[21] J. Kim, K. Kang and J. Lowengrub, Conservative multigrid methods for ternary Cahn-Hilliard systems. Commu. Math. Sci. 2 (2004) 53-77. 
[22] C. Liu and J. Shen, A phase field model for the mixture of two incompressible fluids and its approximation by a fourier-spectral method. Physica D 179 (2003) 211-228.

[23] J.S. Lowengrub and L. Truskinovsky, Quasi-incompressible Cahn-Hilliard fluids and topological transitions. Proc. Royal Soc. London, Ser. A 454 (1998) 2617-2654.

[24] B. Piar, PELICANS: Un outil d'implémentation de solveurs d'équations aux dérivées partielles. Note Technique 2004/33, IRSN (2004)

[25] J.S. Rowlinson and B. Widom, Molecular Theory of Capillarity. Clarendon Press (1982).

[26] K.A. Smith, F.J. Solis and D.L. Chopp, A projection method for motion of triple junctions by level sets. Interfaces and Free Boundaries 4 (2002) 239-261.

[27] R. Temam, Infinite-dimensional dynamical systems in mechanics and physics, Applied Mathematical Sciences 68, SpringerVerlag, New York (1997).

[28] P. Yue, J. Feng, C. Liu and J. Shen, A diffuse-interface method for simulating two-phase flows of complex fluids. J. Fluid Mechanics 515 (2004) 293-317.

To access this journal online:

www.edpsciences.org 